\title{
Chromosome 1q amplification perturbs a ceRNA network to promote melanoma metastasis
}

\section{Xiaonan $\mathrm{Xu}^{1}$, Kaizhen Wang ${ }^{1}$, Olga Vera ${ }^{1}$, Akanksha Verma ${ }^{2}$, Olivier Elemento ${ }^{2}$, Xiaoqing} $\mathrm{Yu}^{3}$, Florian A. Karreth ${ }^{1,4, \#}$

${ }^{1}$ Department of Molecular Oncology, H. Lee Moffitt Cancer Center and Research Institute, Tampa, Florida 33612, USA

2 Caryl and Israel Englander Institute for Precision Medicine, Institute for Computational Biomedicine, Department of Physiology and Biophysics, Weill Cornell Medicine, New York, NY, 10065, USA

${ }^{3}$ Department of Biostatistics and Bioinformatics, H. Lee Moffitt Cancer Center and Research Institute, Tampa, Florida 33612, USA

${ }^{4}$ Lead contact

\# Correspondence: Florian.Karreth@moffitt.org (F.A.K.)

Running title: A ceRNA network promotes melanoma metastasis

Keywords: ceRNA, miRNA, melanoma, metastasis, CEP170, NUCKS1, ZC3H11A, chromosome 1q amplification 


\section{SUMMARY}

Somatic copy number alterations (CNAs) promote cancer, but the underlying driver genes are often not obvious when only the functions of the encoded proteins are considered. mRNAs can act as competitive endogenous miRNA sponges (ceRNAs) to post-transcriptionally regulate gene expression in a protein coding-independent manner. However, whether ceRNAs contribute to the oncogenic effects of CNAs is unknown. We report that chromosome 1q gains promote melanoma progression and metastasis at least in part through overexpression of three mRNAs with ceRNA activity: CEP170, NUCKS1, and ZC3H11A. Genetic studies reveal that these ceRNAs enhance melanoma metastasis by sequestering tumor suppressor miRNAs, thereby alleviating the repression of several pro-metastatic target genes. This regulatory RNA network is evident in other cancer types, suggesting that chromosome $1 \mathrm{q}$ ceRNA deregulation is a common driver of cancer progression. Taken together, our work demonstrates that ceRNAs mediate the oncogenicity of somatic CNAs. 


\section{INTRODUCTION}

Malignant melanoma remains the leading cause of skin cancer-related deaths, almost invariably due to metastases to vital organs. Accordingly, significant efforts have focused on understanding drivers of melanoma progression and metastasis. While genetic events affecting oncogenes and tumor suppressors such as BRAF, NRAS, CDKN2A, and PTEN are welldocumented drivers of melanoma development (Cancer Genome Atlas Network, 2015), surprisingly few metastasis-specific mutations have been discovered (Shain et al., 2018; Vergara et al., 2021). Indeed, mutations and pathways thus far implicated in melanoma metastasis are often those that also promote the early stages of the disease (Damsky et al., 2014; Orgaz and Sanz-Moreno, 2013; Turner et al., 2018), as seen in investigations of autochthonous melanoma models (Ackermann et al., 2005; Cho et al., 2015; Damsky et al., 2011; Dankort et al., 2009; Otsuka et al., 1998; Zingg et al., 2018). Interestingly, copy number alterations (CNA) increase with melanoma progression (Shain et al., 2018; Vergara et al., 2021), suggesting copy number gains and losses as potential drivers of melanoma metastasis. CNA may be focal but can involve entire chromosome arms, obscuring the identity of bona fide metastasis driver genes (Beroukhim et al., 2010). In addition, driver genes are classified based on the function of the encoded protein while potential coding-independent functions of their mRNAs are not usually considered as factors contributing to tumorigenesis.

In addition to being blueprints for translation, mRNAs can act as competitive endogenous RNAs (ceRNAs) that function as natural microRNA (miRNA) sponges. Specifically, ceRNAs competitively bind to miRNAs to impair their repressive activity towards their targets (i.e., ceRNA effectors) (Karreth and Pandolfi, 2013; Salmena et al., 2011). Originally described for pseudogenes (Karreth et al., 2015; Poliseno et al., 2010), ceRNA activity has also been observed for dozens of long noncoding RNAs (IncRNAs) and circular RNAs (circRNAs). Several mRNAs have been reported to promote cancer as ceRNAs, including MYCN (Powers et al., 2016), TYRP1 (Gilot et al., 2017), and several mRNAs that control the expression of PTEN 
(Karreth et al., 2011; Sumazin et al., 2011; Tay et al., 2011). These studies support the notion that mRNAs can function as ceRNAs to regulate gene expression in cancer, yet it is unknown if ceRNA deregulation is a common phenomenon underlying the oncogenic effects of CNAs. We tested whether copy number gains promote melanoma metastasis via overexpression of oncogenic ceRNAs, revealing a chromosome 1q-encoded, pro-metastatic ceRNA network that is controlled by the CEP170, NUCKS1, and ZC3H11A mRNAs. Notably CEP170, NUCKS1, and $Z C 3 H 11 A$ are co-gained in several cancers where their expression correlates with upregulation of pro-metastatic ceRNA effectors, suggesting this potent ceRNA network plays oncogenic roles across cancer types. Our study challenges the notion that somatic CNAs promote cancer predominantly through their encoded proteins and establishes ceRNAs as potent drivers underlying the oncogenicity of somatic CNAs.

\section{RESULTS}

\section{Copy number gains and overexpression of 1q ceRNA genes are associated with melanoma metastasis}

To identify gained/amplified genes with putative ceRNA potential, we analyzed a TCGA Skin Cutaneous Melanoma dataset containing 366 metastatic melanoma cases. Given that gained/amplified genes with high basal expression levels are more likely to elicit prominent ceRNA effects by contributing a large number of miRNA binding sites to their ceRNA networks, we first identified genes as recurrently gained in $\geq 3 \%$ of cases and focused on the top $20 \%$ highest expressed genes. This analysis yielded 211 candidate genes (Table S1). To define putative ceRNA networks we calculated the correlation of the 211 gained candidate genes with the protein-coding transcriptome (20,291 transcripts). We identified 34,285 significant gene pairs with FDR $<0.05$ and Spearman correlation $\geq 0.5$. These gene pairs were then tested for enrichment of known miRNA binding sites based on TargetScan algorithm predictions (release 
7.2) (Agarwal et al., 2015). To capture highly conserved binding sites for the miRNA families (i.e. miRNA Response Elements, MRE), we used the aggregate $P_{C T}$ score (Friedman et al., 2009) with cut-off of $\geq 0.2$ for every predicted miRNA as a stringent probability metric for conserved targeting (Table S2). Out of 34,285 gene pairs, 2,515 gene pairs share at least 4 MREs in their 3'UTRs (Table S3) and could thus constitute a complex ceRNA network encompassing 40 putative ceRNAs and 971 effector transcripts (Figure S1A). The number of effectors for each ceRNA ranged from 1-454 transcripts, with 9 ceRNAs engaging over 100 effectors (Figure 1A).

To further systematically prioritize candidate ceRNAs, we evaluated the connectivity of ceRNA nodes in the network using the Hyperlink-Induced Topic Search (HITS) algorithm (Figure S1B), which revealed the 5 of the top 6 highly ranked putative ceRNA genes located on chromosome 1q (Table S4). The 40 predicted ceRNA genes localize to only 11 genomic regions, with chromosome $6 p$ and $1 q$ harboring 13 and 10 putative ceRNA genes, respectively (Figure S1C). This raised the possibility of co-gained ceRNA genes coordinately regulating the same effectors. ceRNA genes located within a genomic region connect to similar effectors, with the greatest number of shared effectors observed for chromosome 1q ceRNAs (Figure S1D). Among ceRNA genes localized in the chromosome 1q amplicon (1q ${ }^{\text {AMP }}$ ceRNAs), AKT3, NUCKS1, HNRNPU, ZC3H11A, and CEP170 showed the greatest number of total and shared effectors (Figure 1C).

This ceRNA prediction was based on annotated full-length 3'UTRs. However, alternative polyadenylation (APA) can shorten 3'UTRs and thereby limit their ability to sequester miRNAs. Using data from the APAatlas (https://hanlab.uth.edu/apa/) (Hong et al., 2019), we assessed the prevalence of APA across several tissue types for AKT3, NUCKS1, HNRNPU, ZC3H11A, and CEP170. This analysis revealed almost universal APA for HNRNPU, a wide range of polyA site usage for NUCKS1, and predominant usage of canonical polyA sites for $A K T 3, Z C 3 H 11 A$, and CEP170 (Figure S1E). Given shortened 3'UTRs for HNRNPU transcripts, this mRNA was excluded from further analyses. Moreover, there were no significant biological effects of ectopic 
expression of the 3'UTR or coding sequence of $A K T 3$ on two human melanoma cell lines (Figure S1F-H). Consequently, the $1 \mathrm{q}^{\mathrm{AMP}}$ ceRNAs CEP170, NUCKS1, and ZC3H11A were assessed for their roles in melanoma.

Chromosome 1q copy number gains correlate with melanoma stage and occur in over $25 \%$ of metastatic melanoma (Bastian et al., 1998; Fountain et al., 1990; Mertens et al., 1997; Thompson et al., 1995). Accordingly, analysis of a TCGA Skin Cutaneous Melanoma dataset revealed the $1 q^{A M P}$ ceRNA genes CEP170, NUCKS1, and ZC3H11A show co-occurrence of copy number gains or amplification in approximately $52 \%$ (191 out of 367 ) of metastatic melanoma cases (Figure 1D). Further, copy number gains of chromosome 1q are associated with increased expression of CEP170, NUCKS1, and ZC3H11A (Figure S1I) and the expression levels of these 3 genes are significantly correlated (Figure 1E). Expression of CEP170, NUCKS1, and $Z C 3 H 11 A$ is also significantly increased in metastases compared to primary melanomas (Figure 1F). In accord with these findings, CEP170 and NUCKS1 expression is associated with a gene set enrichment analysis metastasis signature, and ZC3H11A also exhibited a trend towards association with the metastasis signature (Figure 1G). Moreover, expression of CEP170, NUCKS1, and ZC3H11A is increased in human melanoma cells compared to human immortalized melanocyte cell lines (Figure 1H, Table S5). Therefore, our data indicate that copy number gains and overexpression of the putative $1 q^{\mathrm{AMP}}$ ceRNAs CEP170, NUCKS1, and ZC3H11A are associated with melanoma progression and metastasis.

\section{$1 q^{A M P}$ ceRNAs have oncogenic effects in melanoma cells in vitro}

To assess the functional potential of CEP170, NUCKS1, and ZC3H11A as ceRNAs, we tested if forced overexpression of their 3'UTRs impacts the phenotype of human melanoma cells. We generated lentiviral vectors that express the 3'UTRs of CEP170, NUCKS1, and ZC3H11A linked to a GFP cDNA to prevent nonsense-mediated decay, ensure proper folding of the 3'UTRs, and enable tracing of cells expressing the 3'UTRs. Ectopic expression of these constructs in the 
human melanoma cell lines A375 and WM793 that lack chromosome 1q CNA resulted in 10-40fold overexpression of the CEP170, NUCKS1, and ZC3H11A 3'UTRs (Figure S2A). While overexpression of the CEP170, NUCKS1, or ZC3H11A 3'UTRs had only modest effects on melanoma cell proliferation (Figure S2B and S2C), there were marked increases in colony number and size under anchorage-independent conditions in soft agar (Figure 2A). Further, overexpression of these 3'UTRs also enhanced melanoma cell migration and invasion in transwell assays (Figure 2B and 2C). Notably, combined overexpression of the CEP170, NUCKS1, and ZC3H11A 3'UTRs further increased anchorage-independent growth, migration, and invasion (Figure 2A-C), suggesting cooperative effects of these ceRNAs.

To affirm the oncogenic potential of $1 q^{A M P}$ ceRNAs, we used shRNAs to silence endogenous CEP170, NUCKS1, or ZC3H11A expression in metastatic 1205Lu human melanoma cells (Figure S2D). Reduced expression of endogenous CEP170, NUCKS1, or ZC3H11A impaired anchorage-independent growth in soft agar, migration and invasion of 1205Lu cells (Figure 2D-F). To test if these effects were mediated by the encoded CEP170, NUCKS1, and ZC3H11A proteins, we overexpressed the coding sequences (CDS) of the $1 q^{\mathrm{AMP}}$ ceRNA genes in A375 and WM793 cells. Interestingly, the CEP170, NUCKS1, and ZC3H11A CDS had no effect on growth in soft agar or on transwell migration and invasion (Figure S2E and 2F), demonstrating that CEP170, NUCKS1, and ZC3H11A augment aggressive melanoma cell phenotypes in vitro in a 3'UTR-dependent but protein coding-independent manner.

\section{$1 q^{A M P}$ ceRNAs augment melanoma metastasis in vivo}

To test the role of the 3'UTRs of CEP170, NUCKS1, and ZC3H11A in promoting melanoma metastasis, A375 and WM793 melanoma cells harboring luciferase were engineered to express the ectopic CEP170, NUCKS1, and ZC3H11A 3'UTRs. A375 cells were subcutaneously injected into the flanks of NSG mice for spontaneous metastasis assays, while WM793 cells were intravenously inoculated into the tail veins of NSG mice. Notably, bioluminescence imaging 
revealed that overexpression of the CEP170, NUCKS1, and ZC3H11A 3'UTRs significantly increased tumor burden in the lungs in the A375 and WM793 metastasis models (Figures 3A and 3B). H\&E staining corroborated the increased lung metastasis burden upon $1 q^{A M P}$ ceRNA overexpression (Figure 3C and 3D). While combined overexpression of the CEP170, NUCKS1, and ZC3H11A 3'UTRs also enhanced lung metastasis, the effect was comparable to overexpression of individual 3'UTRs. Finally, silencing of CEP170, NUCKS1, or ZC3H11A in luciferase-expressing 1205Lu melanoma cells significantly impaired their lung metastatic potential in intravenously inoculated NSG mice (Figure 3E and 3F). Thus, $1 q^{A M P}$ ceRNAs promote the metastatic program of melanoma cells.

\section{$1 q^{A M P}$ ceRNAs sequester tumor suppressive miRNAs}

To define the mechanism underlying the oncogenic effect of the $1 q^{\text {AMP }}$ ceRNAs, we assessed the ability of the CEP170, NUCKS1, and ZC3H11A 3'UTRs to function as miRNA sponges. To this end we created a series of luciferase reporters harboring binding sites for the miRNAs predicted to engage in the $1 q^{\mathrm{AMP}}$ ceRNA network (Table S6). Luc-MRE reporters were expressed in WM793 and A375 melanoma cells with our without the CEP170, NUCKS1, or ZC3H11A 3'UTRs (Figure S3A). This identified miRNAs that are expressed and active (i.e., Luc-MRE reporter activity is reduced compared to empty Luc control reporter) as well as those that are sequestered by the $1 q^{A M P}$ ceRNAs (i.e., Luc-MRE reporter activity is increased by the CEP170, NUCKS1, or ZC3H11A 3'UTRs compared to GFP). This analysis revealed that: (i) miR-135, miR-141, miR-144, miR-200a, and miR-203 are sponged by the CEP170 3'UTR: (ii) miR-135, miR-137, and miR-203 are sponged by the NUCKS1 3'UTR; and (iii) miR-101, miR125, miR-137, miR-144, miR-200bc, and miR-497 are sponged by the ZC3H11A 3'UTR (Figures 4A-C and S3B-D). Of note, miR-135, miR-137, miR-144, and miR-203 are sequestered by two 3'UTRs, and the combined expression of the $1 q^{A M P}$ ceRNA 3'UTRs further enhanced Luciferase activity of these four Luc-MRE reporters (Figure S3E). 
To test direct interaction of these 9 miRNAs with the $1 q^{\mathrm{AMP}}$ ceRNAs, we performed RNA pulldowns using biotinylated probes targeting the GFP sequence in WM793 melanoma cells expressing the CEP170, NUCKS1, or ZC3H11A 3'UTRs (Figure S3A). These analyses revealed that: (i) miR-135, miR-141, miR-144, miR-200a, and miR-203 bound the CEP170 3'UTR; (ii) miR-135, miR-137, and miR-203 bound the NUCKS1 3'UTR; and (iii) miR-101, miR125, miR-137, miR-144, miR-200bc, and miR-497 bound the ZC3H11A 3'UTR (Figure 4D-F). Finally, to further validate the interaction of the $1 q^{A M P}$ ceRNAs with these miRNAs, we transfected miRNA mimics into WM793 melanoma cells. As expected, mimics of: (i) miR-135, miR-141, miR-144, miR-200a, and miR-203 decreased CEP170 expression; (ii) miR-135, miR137, and miR-203 mimics decreased NUCKS1 expression; and (iii) miR-101, miR-125, miR-137, miR-144, miR-200bc, and miR-497 mimics reduced ZC3H11A expression (Figure 4G-I). Thus, the CEP170, NUCKS1, and ZC3H11A 3'UTRs directly bind to and selectively impair the activity of 9 miRNAs.

To assess the stoichiometry of $1 q^{\mathrm{AMP}}$ ceRNAs and the 9 interacting miRNAs, we determined the absolute copy numbers of the endogenous transcripts in A375 and WM793 melanoma cells by Droplet Digital PCR. Notably, with one exception (miR-125) the CEP170, NUCKS1, and ZC3H11A mRNAs are equally or more abundant than these target miRNAs (Figure S3F and S3G). Moreover, with the exception of miR-125, the number of MREs in the $1 \mathrm{q}^{\mathrm{AMP}}$ ceRNAs significantly exceeded the number of molecules of their corresponding miRNAs (Figure $4 \mathbf{J}$ and $\mathbf{4 K}$ ), suggesting that in $1 \mathrm{q}^{\mathrm{AMP}}$ melanoma abundant CEP170, NUCKS1, and ZC3H11A mRNAs can competitively sequester miRNAs.

If overexpression of the $1 q^{A M P}$ ceRNAs reduces the activity of the bound miRNAs toward other targets, these miRNAs may have tumor suppressive potential. To test this, we delivered miRNA mimics and inhibitors to WM793 and 1205Lu melanoma cells and assessed the effects on cell invasion. Notably, 8 out of 9 miRNA mimics reduced cell invasion, and 5 (WM793) and 7 (1205Lu) miRNA inhibitors increased cell invasion to varying extents (Figures 4L, 4M, and 
S3H). Finally, the expression of miR-141, miR-200a/b/c, and miR-203a is reduced in metastatic melanoma compared to primary tumors in a TCGA skin cutaneous melanoma dataset, further supporting tumor suppressor roles for these miRNAs (Figure S3I). These data indicate that $1 \mathrm{q}^{\mathrm{AMP}}$ ceRNAs bind to and inhibit miRNAs having tumor suppressor potential in melanoma.

\section{$1 q^{A M P}$ ceRNAs promote melanoma metastasis in a miRNA-dependent manner}

To test if the oncogenic potential of the $1 q^{A M P}$ ceRNAs is dependent on the interaction with miRNAs, we silenced Dicer in WM793 melanoma cells to acutely inhibit global miRNA biogenesis (Figure S4A). Silencing of Dicer abolished the pro-invasion effects of individual and combined $1 q^{\mathrm{AMP}}$ ceRNA overexpression (Figure 5A). To verify this was through sequestration of the identified tumor suppressor miRNAs, the relevant MREs in the CEP170, NUCKS1, and ZC3H11A 3'UTR overexpression constructs were mutated to prevent miRNA binding to 3'UTRs. Specifically, we mutated: (i) the miR-135, miR-141/200a, miR-144, and miR-203 MREs in CEP170; (ii) the miR-135, miR-137, and miR-203 MREs in NUCKS1; and (iii) the miR-101/144, miR-137, miR-200bc, and miR-497 MREs in ZC3H11A. While overexpression of the wildtype CEP170, NUCKS1, and ZC3H11A 3'UTRs increased the invasion of WM793 and A375 melanoma cells, the MRE-mutant 3'UTRs had no effect (Figures 5B-D and S4B-D). Additionally, the wildtype 3'UTRs but not the MRE-mutant 3'UTRs rescued cell invasion in melanoma cells transfected with the corresponding miRNA mimics (Figures 5B-D and S4B-D). Expression of wildtype but not MRE-mutant CEP170, NUCKS1, or ZC3H11A 3'UTR constructs in $1205 \mathrm{Lu}$ cells in which the corresponding endogenous $1 q^{\mathrm{AMP}}$ ceRNAs were silenced rescued cell invasion in vitro (Figure 5E) and lung metastasis following intravenous transplantation in NSG mice (Figure 5F). These findings demonstrate that the pro-metastatic potential of the $1 \mathrm{q}^{\mathrm{AMP}}$ ceRNAs depends on their ability to sequester their respective tumor suppressor miRNA targets. 
The contribution of miR-101, miR-135, miR-137, miR-141, miR-144, miR-200, miR-203, and miR-497 to the oncogenic effects of endogenously expressed $1 q^{A M P}$ ceRNAs was further tested using miRNA inhibitors and Target Site Blockers (TSBs). Knockdown of CEP170, NUCKS1, or ZC3H11A in 1205Lu melanoma cells reduced the activity of the respective Luc-MRE reporters (Figure 5G-I), indicating that silencing of $1 q^{A M P}$ ceRNAs enhances activity of their miRNA targets. Indeed, co-transfection of the corresponding miRNA inhibitors (i.e., [i] miR-135, miR141, miR-144, miR-200a, or miR-203 inhibitors in CEP170-silenced cells; [ii] miR-135, miR-137, or miR-203 inhibitors in NUCKS1-silenced cells; and [iii] miR-101, miR-137, miR-144, miR$200 \mathrm{bc}$, or miR-497 inhibitors in ZC3H11A-silenced cells) rescued the repression of the Luc-MRE reporters (Figure 5G-I). Similarly, the decrease in cell invasion upon $1 q^{A M P}$ ceRNA silencing was partially rescued by the respective miRNA inhibitors (Figure $\mathbf{5} \mathbf{J}-\mathbf{L}$ ).

TSBs block endogenous ceRNA-miRNA interactions and thereby reduce ceRNA-mediated miRNA sequestration. Delivery of TSBs to $1205 \mathrm{Lu}$ melanoma cells targeting the respective MREs on CEP170, NUCKS1, or ZC3H11A affirmed the miRNA sponge effects of endogenous $1 q^{A M P}$ ceRNAs, where the individual TSBs inhibited invasion of $1205 \mathrm{Lu}$ melanoma cells (Figure S4H-J). To test the effect of blocking the binding of all relevant miRNAs to CEP170, NUCKS1, or ZC3H11A, we delivered combinations of TSBs to $1205 \mathrm{Lu}$ cells. Phenocopying the effects of $1 \mathrm{q}^{\mathrm{AMP}}$ ceRNA silencing, TSBs significantly reduced the invasion of $1205 \mathrm{Lu}$ cells in transwell assays (Figure 5M) and the seeding to the lungs upon intravenous transplantation into NSG mice (Figure $5 \mathrm{~N}$ ). Thus, $1 \mathrm{q}^{\mathrm{AMP}}$ ceRNAs promote melanoma progression and lung metastasis by sequestering 8 tumor suppressive miRNAs.

\section{$1 q^{A M P}$ ceRNAs de-repress pro-metastatic genes by sequestering shared miRNAs.}

A thorough literature search identified 301 metastasis-related genes that are validated targets of the 8 tumor suppressor miRNAs sequestered by the $1 q^{A M P}$ ceRNAs (Figure S5A; Table S7). Among these are 75 genes whose expression significantly correlates (FDR $p<0.0001$, 
Spearman $\geq 0.25$ ) with expression of $1 q^{A M P}$ ceRNAs in the TCGA SKCM dataset (Figure S5B). Moreover, 49 of the 75 putative effectors are significantly overexpressed in melanoma metastases compared to primary tumors (Figure S5C) and 24 of these are shared putative effectors of two or all three $1 q^{A M P}$ ceRNAs (Figure S5D). Protein levels of 7 of these candidate effectors, ZEB1, ZEB2, ROCK1, ROCK2, SP1, SUZ12, and BMI1, increased upon 1q ${ }^{\text {AMP }}$ ceRNA overexpression in WM793 cells (Figures 6A). Moreover, the expression of these putative effectors was suppressed upon silencing of endogenous CEP170, NUCKS1, and ZC3H11A in 1205 Lu melanoma cells (Figure 6B and S5E) and their levels were rescued when the expression of the CEP170, NUCKS1, or ZC3H11A 3'UTRs was restored (Figure 6B).

Notably, the regulation of these putative effectors by $1 q^{A M P}$ ceRNAs was miRNA-dependent. First, transfection of WM793 melanoma cells with the respective miRNA mimics suppressed the expression of the 7 putative effectors (Figure 6C). Second, concomitant overexpression of the CEP170, NUCKS1, or ZC3H11A 3'UTRs limited the repression of these effectors by the miRNA mimics (Figure 6C). Third, transfection of 1205Lu melanoma cells with miRNA inhibitors negated the effects of silencing endogenous CEP170, NUCKS1, or ZC3H11A on the expression of these seven effectors (Figure 6D). Fourth, blocking MREs of endogenous CEP170, NUCKS1, or ZC3H11A mRNAs using TSBs reduced expression of these effectors (Figures 6E and S5F). Finally, individual silencing of the 7 effectors reduced invasion of 1205Lu cells (Figure S5G), indicating their contribution to the phenotype elicited by the $1 \mathrm{q}^{\mathrm{AMP}}$ ceRNAs. Taken together, CEP170, NUCKS1, and ZC3H11A function as ceRNAs that control expression of the prometastatic genes ZEB1, ZEB2, ROCK1, ROCK2, SP1, SUZ12, and BMI1 by sequestering shared miRNAs.

\section{Evidence for the $1 q^{A M P}$ ceRNA network in multiple cancer types}

Chromosome 1q copy number alterations occur in multiple other cancer types (Beroukhim et al., 2010). To investigate the contribution of the $1 q^{\mathrm{AMP}}$ ceRNA network to tumor progression and 
metastasis across cancer types we queried TCGA for concomitant copy number alterations of CEP170, NUCKS1, and ZC3H11A. Significant co-amplifications and co-gains of CEP170, NUCKS1, and ZC3H11A were identified in breast cancer, colon adenocarcinoma, hepatocellular carcinoma, lung adenocarcinoma and lung squamous cell carcinoma (Figure 7A). Notably, the expression of CEP170, NUCKS1, and ZC3H11A strongly correlates with the expression of ZEB1, ZEB2, ROCK1, ROCK2, SP1, SUZ12, and BMI1 (Figure 7B), suggesting the 1q $\mathrm{q}^{\mathrm{AMP}}$ ceRNA network is also functional in these cancer types. Interestingly, a positive correlation between $1 \mathrm{q}^{\mathrm{AMP}}$ ceRNAs and the pro-metastatic effectors ZEB1, ZEB2, ROCK1, ROCK2, SP1, SUZ12, and BMI1 was also evident in some cancers lacking significant alterations of chromosome 1q copy number, including prostate adenocarcinoma, stomach adenocarcinoma, and uveal melanoma (Figure 7B). These findings suggest that a ceRNA network directed by the chromosome 1q CEP170, NUCKS1, and ZC3H11A ceRNAs contributes to the formation and/or progression of several cancer types.

\section{DISCUSSION}

The studies reported herein support a paradigm where chromosome 1q copy number gains promote melanoma progression and metastasis well beyond the consequential increase in expression of certain pro-tumorigenic proteins. Instead, we show that chromosome 1q gains promote melanoma and potentially other cancer types by deregulating an oncogenic ceRNA network controlled by three mRNAs: CEP170, NUCKS1, and ZC3H11A. This oncogenic ceRNA network functions by sequestering 8 tumor suppressive miRNAs, impairing their repression of pro-metastatic target genes and thereby enabling progression to metastatic disease.

The discovery of the ceRNA concept prompted several mathematical models (Ala et al., 2013; Bosia et al., 2013; Chiu et al., 2018; Figliuzzi et al., 2013; Hausser and Zavolan, 2014; Jens and Rajewsky, 2014; Sumazin et al., 2011) and reporter-based quantitative studies 
(Bosson et al., 2014; Denzler et al., 2014, 2016) seeking to understand ceRNA circuits. These studies generally support the possibility of ceRNA-mediated regulation; however, some posit that a ceRNA must add a relatively large number of MREs to a network to elicit physiologically relevant changes in expression. While this contrasts with the extensive experimental evidence supporting ceRNA functions of mammalian mRNAs (Chiu et al., 2017; Gao et al., 2016; Gilot et al., 2017; Jeyapalan et al., 2011; Karreth et al., 2011; Powers et al., 2016; Rutnam and Yang, 2012; Sumazin et al., 2011; Tay et al., 2011), it suggests that specific conditions are required for a mRNA to have ceRNA activity. Thus, the prevalence of ceRNA activity among mRNAs may be moderate. Based on these considerations, we used stringent criteria - high level gains of genes having high basal expression as well as a positive correlation with putative effectors that share binding sites for at least 4 miRNAs - for our ceRNA prediction. Only 40 putative ceRNAs were identified with these criteria, indicating that only a small number of mRNAs may potently contribute as ceRNAs to the oncogenic effects of CNAs in melanoma.

ceRNAs can be classified as exclusive ceRNAs or network ceRNAs based on their mode of action. Exclusive ceRNAs are individual transcripts that sequester a single miRNA family, and unusual circumstances may be required for their activity. For instance, amplification of MYCN in neuroblastoma leads to $>100$-fold overexpression, providing almost $20 \%$ of the let- 7 target site pool, and an MRE:miRNA ratio where MREs significantly exceed the number of let-7 molecules (Powers et al., 2016). Another example of an exclusive ceRNA is TYRP1 that sequesters miR16 in melanoma through three non-canonical target sites that do not provoke TYRP1 mRNA decay (Gilot et al., 2017). By contrast, network ceRNAs, as highlighted by $1 q^{A M P}$ ceRNAs, sequester multiple miRNAs that can converge on the regulation of a biological process such as metastasis. Effective miRNA sequestration can be mediated by the presence of multiple MREs and atypical expression changes or non-canonical MREs may not be strictly required. Indeed, the $1 q^{A M P}$ ceRNAs harbor canonical 8mer and 7mer MREs for the relevant miRNAs and their expression, while more abundant than that of the sequestered miRNAs, does not reach levels 
observed for MYCN. Neither MYCN and TYRP1 (Gilot et al., 2017; Powers et al., 2016) nor the $1 \mathrm{q}^{\mathrm{AMP}}$ ceRNAs (data not shown) reduce miRNA levels, indicating that, given the absence of extensive target site complementarity, target RNA-directed miRNA degradation (TDMD) plays no part in the mechanism of action of these exclusive and network ceRNAs.

The network character of the $1 \mathrm{q}^{\mathrm{AMP}}$ ceRNAs has two intriguing consequences. First, as CEP170, NUCKS1, and ZC3H11A share MREs for the same miRNA families, their overexpression sequesters more copies of these miRNAs. Second, two different ceRNAs can regulate the same effector through different miRNAs. Overall, this raises the ceRNA:miRNA ratio to augment miRNA sequestration. As the robustness of ceRNA crosstalk increases with the number of sequestered miRNAs (Ala et al., 2013; Chiu et al., 2018), concomitant overexpression of $1 q^{A M P}$ ceRNAs may more potently augment the expression of their effectors. Consistent with this notion, combined overexpression of the CEP170, NUCKS1, and ZC3H11A 3'UTRs promotes migration and invasion more potently than overexpression of individual $1 q^{A M P}$ ceRNAs. These findings support the hypothesis that the concerted deregulation of multiple ceRNAs provokes more robust effects (Smillie et al., 2018).

Chromosome 1q gains occur in various cancers in addition to melanoma. Remarkably, we found evidence for the $1 \mathrm{q}^{\mathrm{AMP}}$ ceRNA network in other cancers having $1 \mathrm{q}$ gains and even in cancers in which $1 \mathrm{q}$ gains are rare. This further supports the notion that the $1 \mathrm{q}^{\mathrm{AMP}}$ ceRNA network plays important pathophysiological roles. Besides the 1q ceRNA network, we identified 13 putative ceRNAs localized on chromosome $6 p$. While the $6 p$ ceRNAs are predicted to regulate fewer effectors than the $1 \mathrm{q}$ ceRNAs, they may nevertheless cooperate to promote tumorigenesis in melanomas having $6 p$ gains. Overall, however, ceRNA networks associated with individual CNAs may be rare, at least when our criteria are used for their prediction. Nevertheless, we foresee that additional CNA-associated ceRNA networks contributing to the development of other cancers will be identified, and their importance and oncogenic potential 
will be dictated by the frequency of the CNA and the expression levels of ceRNAs, miRNAs, and effectors.

In summary, we have systematically identified a complex pro-metastatic ceRNA network directly from patient level genomics data and highlight a key regulatory mechanism controlled by three genes, CEP170, NUCKS1, and ZC3H11A, that are concomitantly subjected to copy number alterations. Our study highlights how ceRNA cooperation can strongly distort regulatory networks to drive cancer progression, which we posit is a potential mechanism underlying the oncogenic effects of CNAs. Future studies of CNAs should therefore consider the contribution of ceRNA networks, especially when gains or deletions lack obvious protein-coding driver genes. 


\section{ACKNOWLEDGMENTS}

We are grateful for Karreth lab members for helpful discussions, and to J. Cleveland, A. Gomes, G. DeNicola, and Y. Tay for critical reading of the manuscript. We are grateful to A. Angarita for help with molecular cloning. This work was supported by grants from the National Institutes of Health (R03CA227349, R01CA259046) and from the Melanoma Research Alliance (https://doi.org/10.48050/pc.gr.75702) to F.A.K. This work was also supported by the Gene Targeting Core, which is funded in part by Moffitt's Cancer Center Support Grant (P30CA076292).

\section{AUTHOR CONTRIBUTIONS}

F.A.K conceived and supervised the project. X.X. designed and performed the majority of experiments. K.W. identified validated miRNA targets and O.V. assisted with cell line derivation. A.V., O.E., and X.Y. performed ceRNA network predictions. X.X. and F.A.K. wrote the manuscript with input from all authors.

\section{DECLARATION OF INTERESTS}

The authors declare no competing interests

\section{FIGURE LEGENDS}

Figure 1. Copy number gains and overexpression of predicted 1q ceRNA genes is associated with melanoma metastasis

(A) The number of total predicted effectors for each ceRNA gene. The insert graph shows a close-up of the 21 bottom-ranked ceRNA genes. Predicted ceRNAs encoded by genes localized on chromosome 1q are highlighted in blue. 
(B) The number of putative effectors graphed by the genomic localization of their corresponding ceRNA genes.

(C) Number of unique and shared effectors of the ceRNAs localized on chromosome 1q.

(D) Copy number alterations of CEP170, NUCKS1, and ZC3H11A in TCGA cutaneous melanoma samples. CEP170 and ZC3H11A are gained/amplified in 203 out of 367 samples, while NUCKS1 is gained/amplified in 200 samples. All three genes are co-gained/amplified in 191 samples.

(E) Correlation of CEP170, NUCKS1, and ZC3H11A expression in TCGA cutaneous melanoma samples.

(F) Expression of CEP170, NUCKS1, and ZC3H11A in primary and metastatic cutaneous melanoma samples from TCGA.

(G) GSEA analysis of TCGA cutaneous melanoma samples associates CEP170, NUCKS1, and ZC3H11A expression with a melanoma metastasis gene signature.

(H) Relative CEP170, NUCKS1, and ZC3H11A mRNA levels in human melanocyte (Hermes1, Hermes2, Hermes3A, Hermes4B) and melanoma (WM35, WM115, WM164, WM266.4, 451Lu, 501Mel, SbCl2, SKMel28, A375, WM793, 1205Lu) cell lines.

In $(F, H)$, mean \pm SEM is shown; * $p<0.05,{ }^{* *} p<0.01,{ }^{* * *} p<0.001$.

See also Figure S1.

Figure 2. 1q ${ }^{A M P}$ ceRNAs have oncogenic effects in melanoma cells in vitro

(A) Anchorage-independent growth of A375 and WM793 cells expressing GFP, GFP-CEP1703'UTR, GFP-NUCKS1-3'UTR, GFP-ZC3H11A-3'UTR, or the combination of the three 3'UTRs (Combo). Representative images are show on the left, quantifications are shown on the right.

(B) Quantification of migrated cells in transwell migration assays using the cell lines shown in (A). (C) Quantification of invasive cells in transwell invasion assays using the cell lines shown in (A). (D) Anchorage-independent growth of 1205Lu cells expressing shRNAs targeting CEP170, 
NUCKS1, or ZC3H11A. A scrambled shRNA (sh-Scr) was used as negative control. Representative images are show on the left, quantifications are shown on the right.

(E) Quantification of migrated cells in transwell migration assays using the cell lines shown in (D). (F) Quantification of invasive cells in transwell invasion assays using the cell lines shown in (D).

$\mathrm{N}=3$ biological replicates; two-sided t test. Values represent mean $\pm \mathrm{SEM} ;{ }^{*} \mathrm{p}<0.05,{ }^{* *} \mathrm{p}<$ $0.01,{ }^{* * *} p<0.001$. See also Figure S2.

\section{Figure 3. $1 q^{A M P}$ ceRNAs enhance melanoma metastasis in vivo}

(A-B) Luciferase tagged A375 (A) and WM793 (B) cells expressing GFP, GFP-CEP170-3'UTR, GFP-NUCKS1-3'UTR, GFP-ZC3H11A-3'UTR, or the combination of the three 3'UTRs (Combo) were injected into NSG mice subcutaneously (A375) or intravenously (WM793). Representative bioluminescence images (left) and quantification of the luminescence signals (right) in lungs after 28 days (A375) or 60 days (WM793) are shown.

(C-D) Quantification of lung metastasis burden by H\&E staining of the mice injected with A375 (C) or WM793 (D) cells shown in (A) and (B), respectively.

(D) Quantification of lung metastasis burden by H\&E staining of the mice shown in (B).

(E) Luciferase tagged 1205Lu cells expressing shRNAs against CEP170, NUCKS1, or ZC3H11A or a scrambled control (sh-Scr) were intravenously injected into NSG mice and the luminescence signal in lungs was quantified after 25 days. Representative bioluminescence images (left) and quantification of the luminescence signals (right) are shown.

(F) Quantification of lung metastasis burden by H\&E staining of the mice injected with 1205Lu cells shown in (E).

Each data point in $(A, B, E)$ represents one mouse; $(C, D, F) N=3-4$ biological replicates. Twosided t test; values represent mean \pm SEM. ${ }^{*} p<0.05,{ }^{* *} p<0.01,{ }^{* * *} p<0.001$. 


\section{Figure 4. 1q ${ }^{A M P}$ ceRNAs sequester tumor suppressor miRNAs}

(A) Luciferase assays using dual luciferase reporters harboring MREs for miR-135-5p, miR-1413p, miR-144-3p, miR-200-3p, or miR-203-3p in WM793 cells expressing GFP or GFP-CEP1703'UTR. Luciferase activity was normalized to the reporter without MRE (vector).

(B) Luciferase assays using dual luciferase reporters harboring MREs for miR-135-5p, miR-137, or miR-203-3p in WM793 cells expressing GFP or GFP-NUCKS1-3'UTR. Luciferase activity was normalized to the reporter without MRE (vector).

(C) Luciferase assays using dual luciferase reporters harboring MREs for miR-101-3p, miR-1255p, miR-137, miR-144-3p, miR-200-3p, or miR-497-5p in WM793 cells expressing GFP or GFPZC3H11A-3'UTR. Luciferase activity was normalized to the reporter without MRE (vector). In $(\mathrm{A}-\mathrm{C})$, asterisks $\left({ }^{*},{ }^{* *}\right)$ indicate comparison of Luc-MRE reporter vs. empty control Luc reporter (Vector) and pound symbols $\left({ }^{\#},{ }^{\# \#}\right)$ indicate comparison of 3'UTR vs. GFP.

(D-F) RNA pulldown of GFP-CEP170-3'UTR (D), GFP-NUCKS1-3'UTR (E), or GFP-ZC3H11A3'UTR (F) followed by RT-qPCR for the indicated miRNAs shows the relative enrichment of bound miRNAs. miRNA levels were normalized to pulldown of the GFP control.

(G-I) RT-qPCR showing the relative expression of endogenous CEP170 (G), NUCKS1 $(\mathrm{H})$, and ZC3H11A (I) in 1205Lu cells upon transfection of the indicated miRNA mimics.

$(\mathrm{J}, \mathrm{K})$ Copy number of the indicated mature miRNAs and the corresponding MREs present in endogenous CEP170, NUCKS1, ZC3H11A in A375 (J) and WM793 (K) cells were quantified by Droplet Digital PCR.

(L) Quantification of invasive WM793 cells in transwell invasion assays upon transfection with the indicated miRNA mimics.

(M) Quantification of invasive WM793 cells in transwell invasion assays upon transfection with the indicated miRNA inhibitors.

$\mathrm{N}=3$ biological replicates; two-sided t test. Values represent mean \pm SEM. ${ }^{*} p<0.05,{ }^{* *} p<$ $0.01,{ }^{* * *} p<0.001,{ }^{\#} p<0.05,{ }^{\# \#} p<0.01$. See also Figure S3. 


\section{Figure 5. 1q ${ }^{A M P}$ ceRNA-directed melanoma cell invasion and metastasis is miRNA}

\section{dependent}

(A) Quantification of transwell invasion of WM793 cells overexpressing GFP (gray bars) or the GFP-linked 1 $\mathrm{q}^{\mathrm{AMP}}$ ceRNA 3'UTRs individually or in combination. WM793 cells were transfected with an siRNA pool targeting Dicer or a non-targeting control siRNA pool. Asterisks indicate comparisons of 3'UTR overexpression (light/dark blue bars) vs. control (gray bars) and pound symbol indicates comparison of si-Dicer vs. non-targeting siRNA in vector control samples (gray bars).

(B-D) Quantification of transwell invasion of WM793 cells upon transfection with the indicated miRNA mimics. WM793 cells overexpress GFP, or wildtype or MRE-mutant GFP-CEP1703'UTR (B), GFP-NUCKS1-3'UTR (C), or GFP-ZC3H11A-3'UTR (D). Asterisks indicate comparisons of GFP vs. 3'UTR-WT vs. 3'UTR-Mut and pound symbols indicate comparisons of miRNA mimics vs. scrambled control in GFP control samples (blue bars).

(E) Quantification of transwell invasion of $1205 \mathrm{Lu}$ cells in which the $1 q^{\text {AMP }}$ ceRNAs were knocked-down followed by restoration with either wildtype or MRE-mutant GFP-CEP170-3'UTR, GFP-NUCKS1-3'UTR, or GFP-ZC3H11A-3'UTR.

(F) Luciferase-labeled 1205Lu cells from (E) were intravenously injected into NSG mice and the bioluminescence signal in lungs was quantified after 25 days. In (E-F), asterisks indicate comparisons of control vs. 3'UTR-WT vs. 3'UTR-Mut and pound symbols indicate comparisons of ceRNA shRNAs (light gray bars/dots) vs. control shRNA (dark gray bar/dots).

(G-I) Luciferase assays using 1205Lu cells expressing a control shRNA (sh-Scr) or sh-CEP170 $(\mathrm{G})$, sh-NUCKS1 $(\mathrm{H})$, or sh-ZC3H11A (I). The cells were transfected with the corresponding miRNA inhibitors and MRE-Luc reporters. Luciferase activity normalized to the sh-Scr control is shown. 
(J-L) Quantification of transwell invasion of CEP170- (J), NUCKS1- (K), or ZC3H11A-silenced (L) 1205Lu cells upon transfection with the indicated miRNA inhibitors. Asterisks indicate comparisons of ceRNA shRNA + miRNA inhibitor (blue bars) vs. ceRNA shRNA (light gray bars) and pound symbols indicate comparisons of ceRNA shRNAs (light gray bars) vs. control shRNA (dark gray bars).

(M) Quantification of transwell invasion of $1205 \mathrm{Lu}$ cells transfected with TSB combinations to block all relevant MREs on endogenous CEP170, NUCKS1, or ZC3H11A. Asterisks indicate comparisons of TSBs (light blue bars) vs. control TSB (gray bars).

(N) Luciferase-labeled 1205Lu cells were transfected with TSB combinations to block MREs on endogenous CEP170, NUCKS1, or ZC3H11A followed by intravenous injection into NSG mice. Quantification of the luminescence signal in lungs after 25 days is shown. Asterisks indicate comparisons of TSBs (light blue) vs. control TSB (gray).

$\mathrm{N}=3$ biological replicates; two-sided t test. Values represent mean \pm SEM. ${ }^{*} p<0.05,{ }^{* *} p<$ $0.01,{ }^{* * *} p<0.001,{ }^{\#} p<0.05,{ }^{\# \#} p<0.01,{ }^{\# \#} p<0.001$, ns not significant. See also Figure S4.

Figure 6. 1q ${ }^{A M P}$ ceRNAs de-repress pro-metastatic genes by sequestering shared miRNAs.

(A) Western blot of effector levels upon expression of GFP-CEP170-3'UTR, GFP-NUCKS13'UTR, or GFP-ZC3H11A-3'UTR in WM793 cells.

(B) Western blot of effector levels upon $1 q^{A M P}$ ceRNA silencing in WM793 cells and restoration with GFP-CEP170-3'UTR, GFP-NUCKS1-3'UTR, or GFP-ZC3H11A-3'UTR.

(C) Heatmap showing expression changes of the $1 q^{\text {AMP }}$ ceRNA effectors detected by RT-qPCR. The indicated miRNA mimics were transfected into WM793 cells expressing GFP control, GFPCEP170-3'UTR, GFP-NUCKS1-3'UTR, or GFP-ZC3H11A-3'UTR. Color bar represents $\log _{2}$ fold change of expression. $\log _{2}$ fold change values and $p$-values represented in the heatmap are listed in Table S8 
(D) Heatmap showing expression changes of the $1 q^{\text {AMP }}$ ceRNA effectors detected by RT-qPCR. The indicated miRNA inhibitors were transfected into $1205 \mathrm{Lu}$ cells in which endogenous CEP170, NUCKS1, or ZC3H11A are knocked down. Color bar represents $\log _{2}$ fold change of expression. $\log _{2}$ fold change values and $p$-values represented in the heatmap are listed in Table S9

(E) Western blot of effector levels in1205Lu cells transfected with TSBs combinations to block the relevant MREs on endogenous CEP170, NUCKS1, or ZC3H11A.

$\mathrm{N}=3$ biological replicates. See also Figure S5.

\section{Figure 7. Evidence for the $1 q^{A M P}$ ceRNA network in multiple cancer types}

(A) Venn diagrams showing co-amplifications and co-gains of CEP170, NUCKS1, ZC3H11A in breast cancer (BRCA), colon adenocarcinoma (COAD), hepatocellular carcinoma (HCC), lung adenocarcinoma (LUAD), and lung squamous carcinoma (LUSC) in TCGA datasets.

(B) Heatmaps showing the correlation between $1 \mathrm{q}^{\mathrm{AMP}}$ ceRNAs and effectors in breast cancer (BRCA), colon adenocarcinoma (COAD), hepatocellular carcinoma (HCC), lung adenocarcinoma (LUAD), and lung squamous carcinoma (LUSC), stomach adenocarcinoma (STAD) and uveal melanoma (UVM). Spearman correlation values and $p$-values represented in the heatmap are listed in Table S10 


\section{STAR Methods}

\section{RESOURCE AVAILABILITY}

\section{Lead contact}

Further information and requests for resources and reagents should be directed to and will be fulfilled by the lead contact, Florian A. Karreth (florian.karreth@moffitt.org).

\section{Materials availability}

Plasmids will be deposited at Addgene. Targeted ES cell lines/mouse strains are available upon request from the lead contact.

\section{Data and code availability}

- All data reported in this paper will be shared by the lead contact upon request.

- $\quad$ This paper does not report original code.

- Any additional information required to reanalyze the data reported in this paper is available from the lead contact upon request

\section{EXPERIMENTAL MODEL AND SUBJECT DETAILS}

\section{Cell lines and culture conditions}

Human melanoma cell lines WM793 and 1205Lu were provided by M. Herlyn (Wistar). A375 were obtained from ATCC, HEK293T Lenti-X cells were purchased from Takara. Primary mouse melanoma cell lines were isolated from mouse melanomas. Briefly, tumors were washed in $70 \%$ ethanol and washed in PBS, chopped into small pieces and dissociated using the Milteny Tumor Dissociation Kit (Milteny, \#130-095-929) following the manufacturer's recommendations. Digested tissues were filtered using MACS® SmartStrainers $(70 \mu \mathrm{m})$ (Milteny, \#130-098-462) 
into a conical tube with $5 \mathrm{ml}$ of RPMI-1640 containing 5\% FBS and $1 \%$ Pen/Strep to obtain a single cell suspension. Cells were collected by centrifugation, resuspended in $10 \mathrm{ml}$ of RPMI1640 containing $5 \%$ FBS and $1 \%$ Pen/Strep, and plated in a $10 \mathrm{~cm}$ dish. After $1-2$ passage, cells were transplanted subcutaneously into the flanks of NSG mice for in vivo passaging. Subcutaneous tumors from NSG mice were processed as above and used to establish the murine cell lines. All cell lines were cultured in a humidified atmosphere at $37^{\circ} \mathrm{C}$ and $5 \% \mathrm{CO}_{2}$. Melanoma cell lines were cultured in RPMI-1640 (Lonza) containing 5\% FBS (Sigma). HEK293T Lenti-X were cultured in DMEM (VWR) containing 10\% FBS. All cell lines were confirmed to be mycoplasma-free by the MycoAlert Mycoplasma Detection Kits (Lonza).

\section{Animal models}

All animal experiments were conducted in accordance with an IACUC protocol approved by the University of South Florida. NSG mice were obtained from JAX (Stock No: 005557) and bred inhouse. 6-week-old male and female NSG mice were randomly divided into groups (at least 5 mice per group). $1.5 \times 10^{5} 1205 \mathrm{Lu}$ or $5 \times 10^{5}$ WM793 melanoma cells, were intravenously injected into NSG mice via the tail vein, and lung metastasis burden was monitored after 19-22 days or 50 days, respectively, by IVIS in vivo bioluminescence imaging. $1 \times 10^{5}$ A375 melanoma cells were subcutaneously injected into NSG mice, and spontaneous lung metastases were monitored after 28 days by IVIS in vivo bioluminescence imaging. For IVIS imaging, mice were anesthetized with isoflurane and then intraperitoneally injected with $100 \mu \mathrm{L}$ Luciferin $(4 \mathrm{mg} / \mathrm{ml})$. Bioluminescent imaging was performed 10 min following Luciferin injection using the Xenogen IVIS Spectrum (Caliper Life Sciences). Mice were euthanized 3 days after IVIS imaging, and lungs were resected and fixed in Formalin. Tissue embedding in paraffin, sectioning, and H\&E staining were performed by IDEXX. The quantification of lung metastasis tumor burden was performed with QuPath. ESC-GEMM chimeras were produced as previously described (Bok et al., 2019). Briefly, BP ES cells were targeted with the appropriate targeting vectors via 
recombination-mediated cassette exchange by the Moffitt Gene Targeting Core. Targeting was verified by PCR, and at least two positive clones were injected into Balb/c blastocysts to produce chimeras. Tumor development was induced by administering $25 \mathrm{mg} / \mathrm{ml} 4 \mathrm{OHT}$ in DMSO to the shaved back skin with a paintbrush on 2 consecutive days at 3-4 weeks of age. BP ES cells are male, but the sex of blastocysts at the time of injection is not known. Thus, either male or hermaphroditic chimeras were used. Chimeras were placed on Doxycycline-containing chow (Envigo) immediately following $4 \mathrm{OHT}$ administration. Chimeras were euthanized when primary tumor size reached $1 \mathrm{~mm}^{3}$.

\section{METHOD DETAILS}

\section{TCGA data and ceRNA prediction}

Copy number and expression data from 366 Skin Cutaneous Melanoma (SKCM) metastatic cases in The Cancer Genome Atlas was analyzed. Copy number variation determined by GISTIC 2.0 (-2 = homozygous deletion; -1 = hemizygous deletion; 0 = neutral $/$ no change; $1=$ gain; 2 = high level amplification) was downloaded from cBioPortal (http://www.cbioportal.org/). Gene expression quantified from RNA-seq (TPM values generated by RSEM) was downloaded from Broad Firehose website (https://gdac.broadinstitute.org/). Genes were ranked by average TPM values across 366 metastatic cases. A total of 211 genes with high level amplification in at least $3 \%$ cases and ranked within the top $20 \%$ highest expressed genes were further considered as ceRNA candidates. Spearman correlation was calculated between candidate ceRNAs and 20,291 protein-coding genes. ceRNA-gene pairs with Spearman correlation coefficient $\geq 0.5$ and FDR adjusted P-value $<0.05$ were used for miRNA binding site prediction using TargetScan (Agarwal et al., 2015). A network was constructed with gene pairs sharing at least 4 miRNAs with aggregate $P_{C T}$ score (Friedman et al., 2009) of $\geq 0.2$ using $R$ package network and visualized using $R$ package ggnet2. Connectivity of the ceRNA nodes in the 
network was evaluated using the Hyperlink-Induced Topic Search (HITS) algorithm implemented in R package networkR with default settings.

\section{Gene set enrichment analysis}

Global mRNA expression profiles of TCGA skin cutaneous melanoma dataset (TCGA-SKCM) were subject to GSEA to identify the association of CEP170, NUCKS1, and ZC3H11A with a melanoma metastasis gene signature (WINNEPENNINCKX_MELANOMA_METASTASIS_UP). For GSEA, expression of CEP170, NUCKS1, and ZC3H11A was used as phenotype, and "No_Collapse" was used for gene symbol. The metric for ranking genes in GSEA was set as 'Pearson', otherwise default parameters were used. GSEA was performed using GSEA 4.1.0 software.

\section{Plasmid and lentivirus production}

EGFP in pLenti-GFP-puro was removed with BamHI and Sall and replaced with the open reading frames (ORFs) of human CEP170, NUCKS1, ZC3H11A, or AKT3. The WPRE in pLentiEGFP-blast, pLenti-EGFP-puro, or pLenti-EGFP-hygro was removed with Sall and EcoRI and replaced with the 3'UTRs of CEP170, NUCKS1, ZC3H11A, or AKT3. The sequences of shRNAs targeting CEP170, NUCKS1, or ZC3H11A were obtained from SplashRNA (http://splashrna.mskcc.org/) and cloned into pLenti-EGFP-miRE-puro using Xhol and EcoRI sites. To produce lentivirus supernatants, $6 \mu \mathrm{g}$ of lentiviral vector along with $5.33 \mu \mathrm{g}$ of $\Delta 8.2$ and $0.67 \mu \mathrm{g}$ of VSVG helper plasmid were co-transfected into HEK293T Lenti-X cells in $10 \mathrm{~cm}$ dishes at $90 \%$ confluency. Lentiviral supernatant was collected 48 and 72 hours after tranfection, cleared using a 0.45 micron syringe filter, and used immediately to infect melanoma cells in the presence of $8 \mu \mathrm{g} / \mathrm{ml}$ polybrene or stocked in $-80^{\circ} \mathrm{C}$. Infected cells were selected with the appropriate antibiotics ( $1 \mu \mathrm{g} / \mathrm{ml}$ puromycin, $100 \mu \mathrm{g} / \mathrm{ml}$ hygromycin, or $10 \mu \mathrm{g} / \mathrm{ml}$ blasticidin) for 7 days. 


\section{Proliferation assay}

800 A375 cells/well or 2,000 WM793 cells/well were plated in three 96 well plates in $100 \mu \mathrm{L}$ RPMI-1640 medium containing 5\% FBS. Every other day, one plate was fized and stained cell with $50 \mu \mathrm{L}$ crystal violet solution $(0.5 \%$ crystal violet in $25 \%$ methanol) for 15 minutes. Crystal violet was discarded, and plates were gently washed twice with $\mathrm{ddH}_{2} \mathrm{O}$ and air dried. $150 \mu \mathrm{l}$ of $10 \%$ acetic acid was added per well to extract crystal violet. Absorbance of extracted crystal violet was measured at $600 \mathrm{nM}$ using a Glomax microplate reader.

\section{Soft agar assay}

$1.5 \%$ and $0.8 \%$ SeaPlaque Agarose in PBS solutions were made my microwaving. $1.5 \%$ agarose was mixed at a 1:1 ratio with RPMI-1640 medium to achieve $0.75 \%$ agarose, followed by plating $1 \mathrm{ml}$ per well in a 12 well plate and letting agarose solidify at room temperature. Melanoma cells were trypsinized, counted and 500 cells were resuspended in $500 \mu$ l of RMPI1640 medium containing $20 \%$ FBS. Cells were then mixed with $500 \mu \mathrm{l}$ of $0.8 \%$ agarose $(0.4 \%$ agarose and $10 \%$ FBS final concentrations) and plated atop the solidified $0.75 \%$ agar. Top layer was allowed to solidify at room temperature, followed by culture at $37^{\circ} \mathrm{C}$ with $100 \mu \mathrm{l}$ of fresh medium added every 3 days. Pictures are taken after 10-15 days and analyzed with Image J.

\section{Transwell migration and invasion assay}

To pre-coated transwells with Matrigel, Matrigel was thawed on ice for at least 2 hours, diluted to $5 \%$ with ice-cold basic RPMI-1640 medium, and gently added to transwell inserts (50 $\mu \mathrm{l} / \mathrm{insert)}$ and solidified at $37^{\circ} \mathrm{C}$ for $30 \mathrm{~min}$. Melanoma cells were trypsinized and resuspend in RPMI-1640 medium. For transwell invasion assays, 5,000 A375 cells, 15,000 1205Lu cells, or 30,000 WM793 cells were plated per Matrigel-coated insert in $200 \mu$ l of RPMI-1640 without FBS. For transwell migration assay, 3,000 A375 cells, 10,000 1205Lu cells, or 20,000 WM793 cells were 
plated per insert in $200 \mu \mathrm{l}$ of RPMI-1640 without FBS. $500 \mu \mathrm{l}$ RPMI-1640 medium containing 15\% FBS were added into the bottom well. Plates were incubated at $37^{\circ} \mathrm{C}$ in a humidified incubator for $48 \mathrm{hr}$. Media was discarded, inserts were gently washed once with PBS, and cells were fixed with $500 \mu \mathrm{l}$ fixing solution (ethanol:acetic acid = 3:1) in the bottom well outside the insert for 10 minutes. Inserts were washed once with PBS, and cells were stained in $500 \mu \mathrm{L}$ staining solution ( $0.5 \%$ crystal violet) for 30 minutes. Inserts were washed with tap water twice and non-migrated cells or Matrigel on the top side of the insert were carefully removed with cotton swabs, and inserts were air dried overnight. Pictures were taken at 200x magnification and cell numbers quantified.

\section{miRNA reporter assay}

Reverse complimentary sequences of candidate miRNAs (Table S2) were cloned into psiCHECK2 dual-luciferase reporter vector at the Xhol and Notl sites downstream of Renilla luciferase. $2 \times 10^{4}$ cells/well were seeded in 96-well plates and allowed to settle for 24 hours. For each well of 96 well plate, $15 \mathrm{ng}$ reporter plasmid were transfected using $0.5 \mu \mathrm{l}$ Jetprime in $5 \mu \mathrm{L}$ Jetprime buffer. $48 \mathrm{hr}$ after transfection, cells were washed with PBS and lysed in $50 \mu \mathrm{l}$ of lysis buffer (1x PLB, made from 5x PLB contained in the Dual Luciferase Reporter Assay System kit). Plates were rocked at room temperature for $15 \mathrm{~min}$, and $20 \mu \mathrm{l}$ of cell lysates were transferred to white-walled 96-well plates. Wash the injectors of the Glomax plate reader twice with $\mathrm{ddH}_{2} \mathrm{O}$, prime Injector 1 with buffer LAR II (for Firefly Luciferase) and prime Injector 2 with buffer STOP'n'GLO (for Renilla Luciferase, both contained in the Dual Luciferase Reporter Assay System kit). Set up the procedure for each well (Injector 1 volume: $50 \mu$, waiting duration: 2 seconds, reading integration: 5 seconds, Injector 2 volume: $50 \mu$ l, waiting duration: 2 seconds, reading integration: 5 seconds) then read the plate well by well in the luminometer automatically. Empty psiCHECK-2 control lacking MREs and appropriate vector or scramble controls were included in the experimental setup. Renilla luciferase values were normalized to Firefly 
luciferase value to control for transfection efficiency. To analyze data, normalized Luciferase values of the MRE-bearing reporter were first compared to the empty reporter in vector or scramble control cells, and then the normalized Luciferase values of MRE-bearing reporter were compared to ceRNA overexpression or knockdown conditions.

\section{RNA pulldown}

Biotinylated oligonucleotide probes targeting GFP were designed using the online tool (https://www.biosearchtech.com/support/tools/design-software/chirp-probe-designer) and synthesized by Eton Bioscience. $1 \times 10^{7}$ cells were crosslinked using the UV cross-linker (Spectrolinker) with $600-1200 \mathrm{~J} / \mathrm{cm}^{2}$. Cells were washed with PBS, collected in $1.5 \mathrm{ml}$ tube, and lysed with 500-800 $\mu$ l lysis buffer (TBS (pH7.4-7.6), 1 \% Triton-X100, 0.1-0.5 \% deoxycholate, 0.1-0.5 \% SDS) containing 1\% protease inhibitor cocktail and 0.1-0.5\% RNase inhibitor on ice for $15 \mathrm{~min}$ with repeated vortexing every $5 \mathrm{~min}$. Lysates were cleared by centrifugation at $12,000 \mathrm{~g}$ for $5 \mathrm{~min}$ at $4^{\circ} \mathrm{C}$ and transferred to new $1.5 \mathrm{ml}$ tubes. $100-200 \mu$ of cell lysate were diluted 1:2 in hybridization buffer $(500 \mathrm{mM} \mathrm{NaCl}, 1 \%$ SDS, $100 \mathrm{mM}$ Tris (pH7.0), 10mM EDTA, $15 \%$ formamide). 1-2 nmol of probe mixture was added and mixed by end-to-end rotation at $37^{\circ} \mathrm{C}$ for 4 hours. Streptavidin beads were pre-cleared twice by adding $1 \mathrm{ml}$ of hybridization buffer and collected using a magnetic rack. $20 \mu$ of Streptavidin beads were added per sample, mixed by end-to-end rotation at $37^{\circ} \mathrm{C}$ for 30 minutes, and washed 5 times in wash buffer for 5 minutes each at room temperature. Streptavidin beads were resuspended in $100 \mu$ Proteinase $\mathrm{K}$ buffer (100mM Tris pH7.5, 200mM NaCl, 2mM EDTA, 1\% SDS) containing Proteinase $\mathrm{K}(1$ $\mathrm{mg} / \mathrm{ml}$ ), incubated at $55^{\circ} \mathrm{C}$ for 30 minutes, and boiled for 10 min. RNA was then isolated with the Qiagen miRNeasy kit.

miRNA mimics, miRNA inhibitors, Target Site Blockers (TSB) 
miRNA mimics and miRNA inhibitors were purchased from Horizon Discovery and resuspended in $\mathrm{ddH}_{2} \mathrm{O}$ to prepare $20 \mu \mathrm{M}$ solutions. miRNA mimics or miRNA inhibitors at $10 \mathrm{nM}$ or $20 \mathrm{nM}$ final concentration, respectively, were transfected into melanoma cells using JetPrime according to the manufacturer's recommendations. $48 \mathrm{hr}$ after transfection, transwell invasion assays were performed or RNA was isolated.

Target Site Blockers: TSBs targeting the MREs of (i) miR-135, miR-141/200a, miR-144, or miR203 on the CEP170 3'UTR; (ii) miR-135, miR-137, or miR-203 on the NUCKS1 3'UTR; and (iii) miR-101/137/144, or miR-200b/200c on the ZC3H11A 3'UTR were obtained from Qiagen. TSBs were resuspended in $\mathrm{dd}_{2} \mathrm{O}$ to prepare a $50 \mu \mathrm{M}$ solution. TSBs were transfected into melanoma cells at a final concentration of 50nM. For xenograft metastasis assays, melanoma cells were intravenously injected into NSG mice $24 \mathrm{hr}$ after TSB transfection. Transwell invasion assays were performed $48 \mathrm{hr}$ after TSB transfection. RNA and protein were isolated $72 \mathrm{hr}$ and $96 \mathrm{hr}$, respectively, after TSB transfection.

\section{Droplet digital PCR (ddPCR)}

RNA was isolated from $1 \times 10^{6}$ cells using Trizol using the manufacturer's recommendation. RNA was reverse transcribed into cDNA using the TaqMan MicroRNA Reverse Transcription Kit (Thermo Fisher Scientific) (miRNA) or PrimeScrip RT Master Mix (Takara) (mRNA). cDNA samples (10fg 100ng/reaction), Taqman probes (FAM, 20x), ddPCR supermix (2x) and dd $\mathrm{H}_{2} \mathrm{O}$ were combined in a total volume of $22 \mu$ in 96 -well plates, and plates were sealed, centrifuged for $30 \mathrm{sec}$ at 1,000rpm, and loaded into the Generator along with required consumables (DG32 cartridges, tips, waste bins, droplet plate, and oil) to partition the samples into droplets. Plates the containing droplets were sealed with foil, and PCR was performed to end point ( 40 cycles) using the conditions outlined in Table S11. Plates were then loaded into the QX200 Reader to quantify positive and negative droplets in each sample and to plot fluorescence signal droplet by 
droplet. Results are visualized in Quantasoft Software. The fraction of positive droplets in a sample determines the concentration of target in copies/ $\mu$.

\section{$R T-q P C R$}

RNA was extracted from cells using TRIzol (Invitrogen) following protocols supplied by the manufacturer. First-strand cDNA was generated with PrimeScript RT Master Mix (Takara). qPCR was performed on StepOnePlus ${ }^{\mathrm{TM}}$ Real-Time PCR System (Thermo Fisher), and PerfeCTa SYBR green Fastmix (Quantabio) was used for gene detection. The sequences of primers were obtained from PrimerBank (https://pga.mgh.harvard.edu/primerbank/index.html) and are listed in Table S2.

\section{Western blot}

$15 \mu \mathrm{g}$ of protein were loaded onto NuPAGE 4\%-12\% precast gels and separated by electrophoresis with NuPAGE running buffer at constant $120 \mathrm{~V}$ for approximately $2 \mathrm{hr}$. Proteins were transferred to nitrocellulose membranes with transfer buffer at constant $300 \mathrm{~mA}$ for $2 \mathrm{hr}$. Membranes were blocked in 5\% non-fat dry milk solution in TBST for 30 min and then incubated with one of the following primary antibodies overnight at $4^{\circ} \mathrm{C}$ : anti-ZEB1 $(1: 1,000)$, anti-ZEB2 (1:1,000), anti-ROCK1 (1:1,000), anti-ROCK2 $(1: 1,000)$, anti-SP1 $(1: 1,000)$, anti-SUZ12 $(1: 1,000)$, or anti-BMl1 $(1: 1,000)$. Anti-beta-actin $(1: 3,000)$ was blotted as a loading control. Membranes were washed 3 times with TBST for $10 \mathrm{~min}$, followed by incubation with HRPconjugated secondary antibodies $(1: 2,000)$ for $1 \mathrm{hr}$ room temperature. After three washes in TBST, chemiluminescence substrate (1:1) was applied to the blot for 4 min and chemiluminescence signal was captured using a LI-COR imaging system.

\section{Quantification and statistical analysis}


Proliferation assays are presented as mean \pm SD. For soft agar, transwell migration, and invasion assays, 3 or 4 random fields were quantified for each well and data are presented as mean \pm SEM. For luciferase reporter assays and RNA pulldown assays data are presented as mean \pm SEM. Droplet digital PCR data were generated using 4 replicates and presented as mean \pm SD. All experiments were performed at least 3 times with 3-4 technical replicates. Statistical analyses were performed by Student's unpaired t test. Data of in vivo imagining of lung metastasis are presented as mean \pm SEM. Survival analysis of ESC-GEMMs was based on a cohort of 58 mice (22 TRE-GFP mice, 10 TRE-CEP170 mice, 11 TRE-NUCKS1 mice, and 15 TRE-ZC3H11A mice) and the $p$ values were calculated based on a log-rank test. A p value of $<0.05$ was considered statistically significant. 


\section{SUPPLEMENTAL TABLES}

Supplemental Table S1: List of 211 candidate ceRNAs

Supplemental Table S2: List of miRNA families predicted for the candidate ceRNAs

Supplemental Table S3: List of ceRNA/effector gene pairs

Supplemental Table S4: HITS analysis

Supplemental Table S5: Expression of ceRNAs in melanocyte and melanoma cell lines

Supplemental Table S6: Luc-MRE reporter assay results for sponging of predicted miRNAs

Supplemental Table S7: List of validated pro-metastatic targets of sponged miRNAs

Supplemental Table S8: $\log _{2}$ fold change of expression of effectors upon ceRNA 3'UTR overexpression \pm miRNA mimics

Supplemental Table S9: $\log _{2}$ fold change of expression of effectors upon ceRNA silencing \pm miRNA inhibitors

Supplemental Table S10: Spearman correlations of $1 q^{A M P}$ ceRNAs and effectors across 8 cancer types

Supplemental Table S11: RT-qPCR primer sequences, MRE reporter sequences, transfection conditions, ddPCR procedure 


\section{SUPPLEMENTAL FIGURE LEGENDS}

Figure S1. CEP170, NUCKS1, and ZC3H11A, but not AKT3 and HNRNPU, are potential ceRNAs on chromosome 1q, related to Figure 1

(A) The metastatic melanoma ceRNA network. Central triangles represent gained/amplified ceRNA genes and peripheral circles represent putative effectors.

(B) HITS score of the 40 predicted ceRNAs in melanoma. The insert graph shows a close-up of the 25 bottom-ranked ceRNA genes. Predicted ceRNAs encoded by genes localized on chromosome 1q are highlighted in blue.

(C) The number of predicted ceRNA genes by genomic localization.

(D) The number of shared effectors of the 40 predicted ceRNAs in melanoma. The insert graph shows a close-up of the 22 bottom-ranked ceRNA genes. Predicted ceRNAs encoded by genes localized on chromosome 1q are highlighted in blue.

(E) Percentage Distal Usage Index (PDUI) scores of AKT3, NUCKS1, HNRNPU, ZC3H11A, and CEP170, the top ranked ceRNAs localized on chromosome 1q.

(F) Proliferation assay of A375 and WM793 cells overexpressing GFP-AKT3-3'UTR, the AKT3 coding sequence (CDS) or a GFP control.

(G) Anchorage-independent growth in soft agar of A375 and WM793 cells overexpressing the GFP-AKT3-3'UTR or a GFP control.

(H) Migration in a transwell assay of A375 and WM793 cells overexpressing the GFP-AKT33'UTR or a GFP control.

(I) Correlation between genomic alteration and gene expression of CEP170, NUCKS1, and ZC3H11A.

In $(F-H), N=3$ biological replicates; two-sided t test. Values represent mean \pm SEM. ** $p<0.01$, ns not significant. 
Figure S2. Quantification of $1 q^{A M P}$ ceRNA overexpression and silencing, and effect of $1 q^{A M P}$ ceRNA CDS on melanoma cell biology, related to Figure 2

(A) RT-qPCR showing the relative levels of the $1 q^{\text {AMP }}$ ceRNA 3'UTRs in WM793 and A375 cells expressing GFP-CEP170-3'UTR, GFP-NUCKS1-3'UTR, or GFP-ZC3H11A-3'UTR. Expression was normalized to the GFP control cells.

(B) Proliferation assays of A375 cells expressing GFP, the $1 q^{A M P}$ ceRNA 3'UTRs (GFPCEP170-3'UTR, GFP-NUCKS1-3'UTR, or GFP-ZC3H11A-3'UTR), or the 1q ${ }^{A M P}$ ceRNA CDS.

(C) Proliferation assays of WM793 cells expressing GFP, the $1 q^{\text {AMP }}$ ceRNA 3'UTRs (GFPCEP170-3'UTR, GFP-NUCKS1-3'UTR, or GFP-ZC3H11A-3'UTR), or the 1q ${ }^{\text {AMP }}$ ceRNA CDS.

(D) RT-qPCR showing the knock down efficiency of CEP170, NUCKS1, or ZC3H11A in 1205Lu cells.

(E) Quantification of anchorage-independent growth in soft agar of A375 and WM793 cells overexpressing the $1 q^{\mathrm{AMP}}$ ceRNA CDS.

(F) Quantification of transwell migration assay of A375 and WM793 cells overexpressing the $1 q^{\text {AMP }}$ ceRNA CDS.

$\mathrm{N}=3$ biological replicates; two-sided t test. Values represent mean \pm SEM. ${ }^{*} p<0.05,{ }^{* *} p<$ $0.01,{ }^{* * *} p<0.001$, ns not significant.

Figure S3. Identification of tumor suppressive miRNAs that are sequestered by the $1 \mathrm{q}^{\mathrm{AMP}}$ ceRNAs, related to Figure 4

(A) (i)Outline of the MRE luciferase reporter assay. MREs were cloned downstream of Renilla luciferase, leading to repression by endogenous miRNAs. Co-expression of $1 q^{A M P}$ ceRNAs will result in de-repression of the reporter constructs. (ii) Outline of the RNA pulldown assay. Biotinylated GFP probes were used to pull down GFP, GFP-CEP170-3'UTR, GFP-NUCKS13'UTR, and GFP-ZC3H11A-3'UTR with Streptavidin beads and bound miRNAs were detected by RT-qPCR. 
(B) Luciferase assays using dual luciferase reporters harboring MREs for miR-135-5p, miR-1413p, miR-144-3p, miR-200-3p, or miR-203-3p in A375 cells expressing GFP or GFP-CEP1703'UTR. Luciferase activity was normalized to the reporter without MRE (vector).

(C) Luciferase assays using dual luciferase reporters harboring MREs for miR-135-5p, miR-137, or miR-203-3p in A375 cells expressing GFP or GFP-NUCKS1-3'UTR. Luciferase activity was normalized to the reporter without MRE (vector).

(D) Luciferase assays using dual luciferase reporters harboring MREs for miR-101-3p, miR-1255p, miR-137, miR-144-3p, miR-200-3p, or miR-497-5p in A375 cells expressing GFP or GFPZC3H11A-3'UTR. Luciferase activity was normalized to the reporter without MRE (vector). In (B-D), asterisks $\left({ }^{*},{ }^{* *}\right)$ indicate comparison of Luc-MRE reporter vs. empty control Luc reporter (Vector) and pound symbols $\left({ }^{\#},{ }^{\# \#}\right)$ indicate comparison of 3'UTR vs. GFP.

(E) Luciferase assays using dual luciferase reporters harboring MREs for miR-135-5p, miR-137, miR-144-3p, or miR-200-3p in WM793 cells expressing the $1 q^{\text {AMP }}$ ceRNA 3'UTRs individually or in combination. Luciferase activity was normalized to the reporter without MRE (vector), not shown.

(F-G) Copies of the indicated mature miRNAs and endogenous CEP170, NUCKS1, ZC3H11A per ng RNA were quantified by Droplet Digital PCR in A375 (F) and WM793 (G) cells.

(H) Quantification of transwell invasion assays with 1205Lu cells transfected with the indicated miRNA inhibitors.

(I) Expression of the indicated miRNAs in primary and metastatic melanoma samples in the TCGA melanoma dataset.

In $(B-D, H), N=3$ biological replicates. Two-sided t test; values represent mean $\pm S E M .{ }^{*} p<$ $0.05,{ }^{* *} p<0.01,{ }^{* \star *} p<0.001,{ }^{\#} p<0.05,{ }^{\# \#} p<0.01$.

Figure S4. The oncogenic effect of the $1 q^{A M P}$ ceRNAs depends on miRNA binding, related to Figure 5 
(A) Western blot validates the siRNA-mediated silencing of Dicer in $1205 \mathrm{Lu}$ cells expressing $1 q^{\text {AMP }}$ ceRNAs.

(B-D) Quantification of transwell invasion of A375 cells upon transfection with the indicated miRNA mimics. A375 cells were engineered to overexpress GFP, or wildtype or MRE-mutant GFP-CEP170-3'UTR (B), GFP-NUCKS1-3'UTR (C), or GFP-ZC3H11A-3'UTR (D). Asterisks indicate comparisons of GFP vs. 3'UTR-WT vs. 3'UTR-Mut and pound symbols indicate comparisons of miRNA mimics vs. scrambled control in GFP control samples (blue bars).

(E-G) RT-qPCR to assess restoration of $1 q^{A M P}$ ceRNA expression upon wildtype or MRE-mutant GFP-CEP170-3'UTR (E), GFP-NUCKS1-3'UTR (F), or GFP-ZC3H11A-3'UTR (G) expression in 1205Lu cells having silenced endogenous CEP170, NUCKS1, or ZC3H11A, respectively. Asterisks indicate comparisons of 3'UTR expression in 3'UTR-WT/Mut restored vs. ceRNA shRNA and pound symbols indicate comparison of ceRNA shRNA vs. shRNA control.

(H-J) Quantification of transwell invasion of 1205Lu cells transfected with the indicated target site blockers (TSBs) to block MREs on endogenous CEP170 (H), NUCKS1 (I), or ZC3H11A (J). Asterisks indicate comparisons of TSBs (light blue bars) vs. control TSB (gray bars).

$\mathrm{N}=3$ biological replicates; two-sided t test. Values represent mean \pm SEM. ${ }^{*} p<0.05,{ }^{* *} p<$ $0.01,{ }^{* * *} p<0.001,{ }^{\#} p<0.05,{ }^{\# \#} p<0.01,{ }^{\# \#} p<0.001$, ns not significant.

Figure S5. The $1 q^{A M P}$ ceRNAs regulate expression of pro-metastatic effectors, related to Figure 6

(A) 301 metastasis associated genes are validated targets of one or more of the miRNAs sequestered by the $1 \mathrm{q}^{\mathrm{AMP}}$ ceRNAs, based on published literature.

(B) The expression correlation between the 301 miRNA targets and CEP170, NUCKS1, or ZC3H11A in the TCGA cutaneous melanoma dataset are shown. 72 genes are highly correlated $(R>0.25)$ and are potential ceRNA effectors. 
(C) Volcano plot showing the differential expression of potential ceRNA effectors in primary and metastatic melanoma samples in TCGA dataset. 49 potential effectors are significantly higher expressed in metastatic melanoma compared to primary melanoma.

(D) Venn diagram showing that expression of 24 out of 49 putative effector correlates with two or three of the $1 q^{A M P}$ ceRNAs.

(E) RT-qPCR showing expression of the indicated effectors in 1205Lu melanoma cells upon silencing endogenous CEP170, NUCKS1, or ZC3H11A. Effector expression was normalized to cells expressing a scrambled control shRNA (sh-Scr).

(F) Western blot showing the protein expression of the indicated effectors in 1205Lu melanoma cells following treatment with individual TSBs.

(G) Quantification of invasion of $1205 \mathrm{Lu}$ cells upon silencing of the indicated effectors with siRNAs. Values represent mean \pm SEM. ${ }^{* *} p<0.001$ 


\section{REFERENCES}

Ackermann, J., Frutschi, M., Kaloulis, K., McKee, T., Trumpp, A., and Beermann, F. (2005). Metastasizing melanoma formation caused by expression of activated N-RasQ61K on an INK4a-deficient background. Cancer Research 65, 4005-4011.

Agarwal, V., Bell, G.W., Nam, J.-W., and Bartel, D.P. (2015). Predicting effective microRNA target sites in mammalian mRNAs. ELife 4, 101.

Ala, U., Karreth, F.A., Bosia, C., Pagnani, A., Taulli, R., Léopold, V., Tay, Y., Provero, P., Zecchina, R., and Pandolfi, P.P. (2013). Integrated transcriptional and competitive endogenous RNA networks are cross-regulated in permissive molecular environments. Proceedings of the National Academy of Sciences of the United States of America 110, 7154-7159.

Bastian, B.C., LeBoit, P.E., Hamm, H., Bröcker, E.B., and Pinkel, D. (1998). Chromosomal gains and losses in primary cutaneous melanomas detected by comparative genomic hybridization. Cancer Research $58,2170-2175$.

Beroukhim, R., Mermel, C.H., Porter, D., Wei, G., Raychaudhuri, S., Donovan, J., Barretina, J., Boehm, J.S., Dobson, J., Urashima, M., et al. (2010). The landscape of somatic copy-number alteration across human cancers. Nature 463, 899-905.

Bok, I., Vera, O., Xu, X., Jasani, N., Nakamura, K., Reff, J., Nenci, A., Gonzalez, J.G., and Karreth, F.A. (2019). A versatile ES cell-based melanoma mouse modeling platform. Cancer Research 80, canres.2924.2019-921.

Bosia, C., Pagnani, A., and Zecchina, R. (2013). Modelling Competing Endogenous RNA Networks. PloS One 8 , e66609.

Bosson, A.D., Zamudio, J.R., and Sharp, P.A. (2014). Endogenous miRNA and Target Concentrations Determine Susceptibility to Potential ceRNA Competition. Molecular Cell 56, 347-359.

Chiu, H.-S., Martínez, M.R., Bansal, M., Subramanian, A., Golub, T.R., Yang, X., Sumazin, P., and Califano, A. (2017). High-throughput validation of ceRNA regulatory networks. Bmc Genomics 18, 418.

Chiu, H.-S., Martínez, M.R., Komissarova, E.V., Llobet-Navas, D., Bansal, M., Paull, E.O., Silva, J., Yang, X., Sumazin, P., and Califano, A. (2018). The number of titrated microRNA species dictates ceRNA regulation. Nucleic Acids Research 136, 215-4369.

Cho, J.H., Robinson, J.P., Arave, R.A., Burnett, W.J., Kircher, D.A., Chen, G., Davies, M.A., Grossmann, A.H., VanBrocklin, M.W., McMahon, M., et al. (2015). AKT1 Activation Promotes Development of Melanoma Metastases. Cell Reports 13, 898-905.

Damsky, W.E., Curley, D.P., Santhanakrishnan, M., Rosenbaum, L.E., Platt, J.T., Rothberg, B.E.G., Taketo, M.M., Dankort, D., Rimm, D.L., McMahon, M., et al. (2011). $\beta$-catenin signaling controls metastasis in Braf-activated Pten-deficient melanomas. Cancer Cell 20, 741-754. 
Damsky, W.E., Theodosakis, N., and Bosenberg, M. (2014). Melanoma metastasis: new concepts and evolving paradigms. Oncogene 33, 2413-2422.

Dankort, D., Curley, D.P., Cartlidge, R.A., Nelson, B., Karnezis, A.N., Damsky, W.E., You, M.J., DePinho, R.A., McMahon, M., and Bosenberg, M. (2009). Braf(V600E) cooperates with Pten loss to induce metastatic melanoma. Nature Genetics 41, 544-552.

Denzler, R., Agarwal, V., Stefano, J., Bartel, D.P., and Stoffel, M. (2014). Assessing the ceRNA hypothesis with quantitative measurements of miRNA and target abundance. Molecular Cell 54, 766-776.

Denzler, R., McGeary, S.E., Title, A.C., Agarwal, V., Bartel, D.P., and Stoffel, M. (2016). Impact of MicroRNA Levels, Target-Site Complementarity, and Cooperativity on Competing Endogenous RNARegulated Gene Expression. Molecular Cell 64, 565-579.

Figliuzzi, M., Marinari, E., and Martino, A.D. (2013). MicroRNAs as a selective channel of communication between competing RNAs: a steady-state theory. Biophysical Journal 104, 1203-1213.

Fountain, J.W., Bale, S.J., Housman, D.E., and Dracopoli, N.C. (1990). Genetics of melanoma. Cancer Surveys $9,645-671$.

Friedman, R.C., Farh, K.K.-H., Burge, C.B., and Bartel, D.P. (2009). Most mammalian mRNAs are conserved targets of microRNAs. Genome Research 19, 92-105.

Gao, S., Cheng, C., Chen, H., Li, M., Liu, K., and Wang, G. (2016). IGF1 3'UTR functions as a ceRNA in promoting angiogenesis by sponging miR-29 family in osteosarcoma. J Mol Histol 47, 135-143.

Gilot, D., Migault, M., Bachelot, L., Journé, F., Rogiers, A., Donnou-Fournet, E., Mogha, A., Mouchet, N., Pinel-Marie, M.-L., Mari, B., et al. (2017). A non-coding function of TYRP1 mRNA promotes melanoma growth. Nature Cell Biology 19, 1348-1357.

Hausser, J., and Zavolan, M. (2014). Identification and consequences of miRNA-target interactions-beyond repression of gene expression. Nature Reviews. Genetics 15, 599-612.

Hong, W., Ruan, H., Zhang, Z., Ye, Y., Liu, Y., Li, S., Jing, Y., Zhang, H., Diao, L., Liang, H., et al. (2019). APAatlas: decoding alternative polyadenylation across human tissues. Nucleic Acids Res 48, D34-D39.

Jens, M., and Rajewsky, N. (2014). Competition between target sites of regulators shapes posttranscriptional gene regulation. Nature Reviews. Genetics.

Jeyapalan, Z., Deng, Z., Shatseva, T., Fang, L., He, C., and Yang, B.B. (2011). Expression of CD44 3'untranslated region regulates endogenous microRNA functions in tumorigenesis and angiogenesis.

Nucleic Acids Res 39, 3026-3041.

Karreth, F.A., and Pandolfi, P.P. (2013). ceRNA cross-talk in cancer: when ce-bling rivalries go awry. Cancer Discovery 3,1113-1121. 
Karreth, F.A., Tay, Y., Perna, D., Ala, U., Tan, S.M., Rust, A.G., DeNicola, G., Webster, K.A., Weiss, D., Perez-Mancera, P.A., et al. (2011). In vivo identification of tumor- suppressive PTEN ceRNAs in an oncogenic BRAF-induced mouse model of melanoma. Cell 147, 382-395.

Karreth, F.A., Reschke, M., Ruocco, A., Ng, C., Chapuy, B., Léopold, V., Sjoberg, M., Keane, T.M., Verma, A., Ala, U., et al. (2015). The BRAF pseudogene functions as a competitive endogenous RNA and induces lymphoma in vivo. Cell 161,319-332.

Mertens, F., Johansson, B., Höglund, M., and Mitelman, F. (1997). Chromosomal imbalance maps of malignant solid tumors: a cytogenetic survey of 3185 neoplasms. Cancer Research 57, 2765-2780.

Network, C.G.A. (2015). Genomic Classification of Cutaneous Melanoma. Cell 161, 1681-1696.

Orgaz, J.L., and Sanz-Moreno, V. (2013). Emerging molecular targets in melanoma invasion and metastasis. Pigment Cell \& Melanoma Research 26, 39-57.

Otsuka, T., Takayama, H., Sharp, R., Celli, G., LaRochelle, W.J., Bottaro, D.P., Ellmore, N., Vieira, W., Owens, J.W., Anver, M., et al. (1998). c-Met autocrine activation induces development of malignant melanoma and acquisition of the metastatic phenotype. Cancer Research 58, 5157-5167.

Poliseno, L., Salmena, L., Zhang, J., Carver, B., Haveman, W.J., and Pandolfi, P.P. (2010). A codingindependent function of gene and pseudogene mRNAs regulates tumour biology. Nature 465, 1033-1038.

Powers, J.T., Tsanov, K.M., Pearson, D.S., Roels, F., Spina, C.S., Ebright, R., Seligson, M., Soysa, Y. de, Cahan, P., Theißen, J., et al. (2016). Multiple mechanisms disrupt the let-7 microRNA family in neuroblastoma. Nature 535, 246-251.

Rutnam, Z.J., and Yang, B.B. (2012). The non-coding 3' UTR of CD44 induces metastasis by regulating extracellular matrix functions. J Cell Sci 125, 2075-2085.

Salmena, L., Poliseno, L., Tay, Y., Kats, L., and Pandolfi, P.P. (2011). A ceRNA hypothesis: the Rosetta Stone of a hidden RNA language? Cell 146, 353-358.

Shain, A.H., Joseph, N.M., Yu, R., Benhamida, J., Liu, S., Prow, T., Ruben, B., North, J., Pincus, L., Yeh, I., et al. (2018). Genomic and Transcriptomic Analysis Reveals Incremental Disruption of Key Signaling Pathways during Melanoma Evolution. Cancer Cell 34, 45-55.e4.

Smillie, C.L., Sirey, T., and Ponting, C.P. (2018). Complexities of post-transcriptional regulation and the modeling of ceRNA crosstalk. Critical Reviews in Biochemistry and Molecular Biology 53, 1-15.

Sumazin, P., Yang, X., Chiu, H.-S., Chung, W.-J., Iyer, A., Llobet-Navas, D., Rajbhandari, P., Bansal, M., Guarnieri, P., Silva, J., et al. (2011). An extensive microRNA-mediated network of RNA-RNA interactions regulates established oncogenic pathways in glioblastoma. Cell 147, 370-381.

Tay, Y., Kats, L., Salmena, L., Weiss, D., Tan, S.M., Ala, U., Karreth, F., Poliseno, L., Provero, P., Cunto, F.D., et al. (2011). Coding-independent regulation of the tumor suppressor PTEN by competing endogenous mRNAs. Cell 147, 344-357. 
Thompson, F.H., Emerson, J., Olson, S., Weinstein, R., Leavitt, S.A., Leong, S.P., Emerson, S., Trent, J.M., Nelson, M.A., and Salmon, S.E. (1995). Cytogenetics of 158 patients with regional or disseminated melanoma. Subset analysis of near-diploid and simple karyotypes. Cancer Genetics and Cytogenetics 83, 93-104.

Turner, N., Ware, O., and Bosenberg, M. (2018). Genetics of metastasis: melanoma and other cancers. Clinical \& Experimental Metastasis 35, 379-391.

Vergara, I.A., Mintoff, C.P., Sandhu, S., McIntosh, L., Young, R.J., Wong, S.Q., Colebatch, A., Cameron, D.L., Kwon, J.L., Wolfe, R., et al. (2021). Evolution of late-stage metastatic melanoma is dominated by aneuploidy and whole genome doubling. Nat Commun 12, 1434.

Zingg, D., Debbache, J., Peña-Hernández, R., Antunes, A.T., Schaefer, S.M., Cheng, P.F., Zimmerli, D., Haeusel, J., Calçada, R.R., Tuncer, E., et al. (2018). EZH2-Mediated Primary Cilium Deconstruction Drives Metastatic Melanoma Formation. Cancer Cell 34, 69-84.e14. 
A

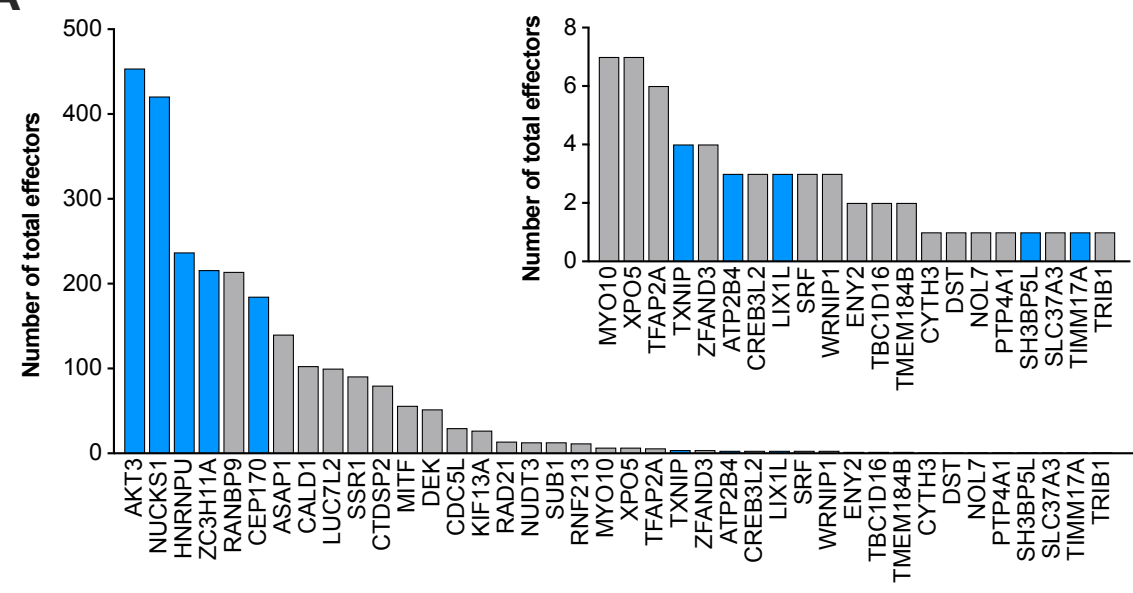

B

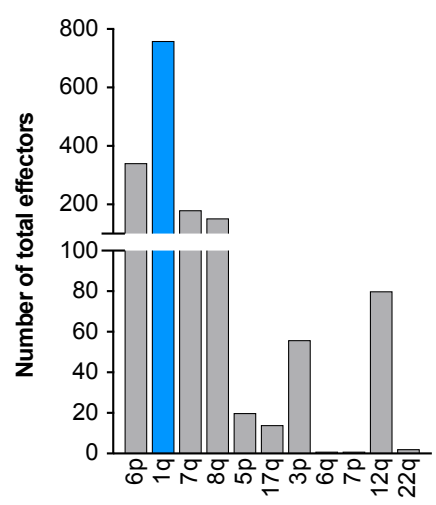

C

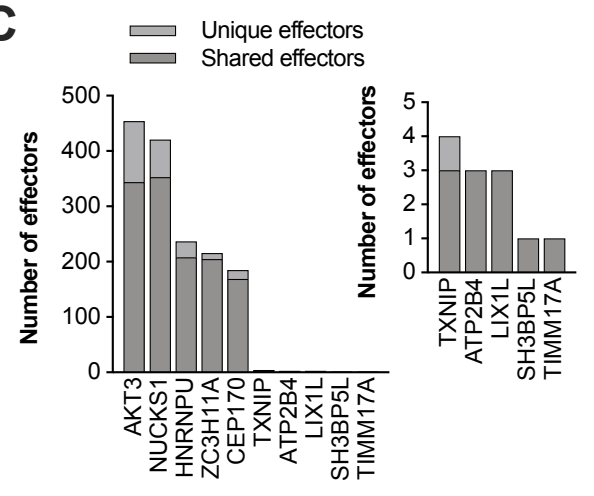

F

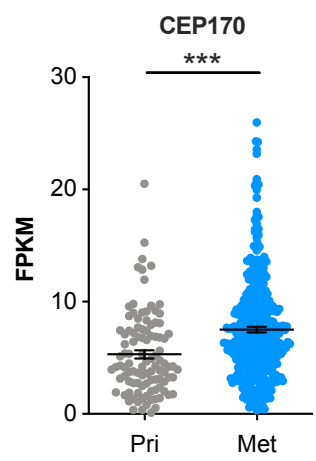

H

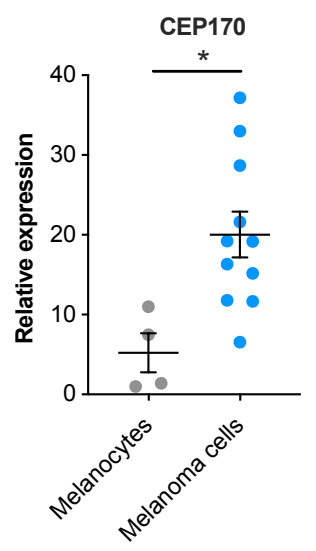

D

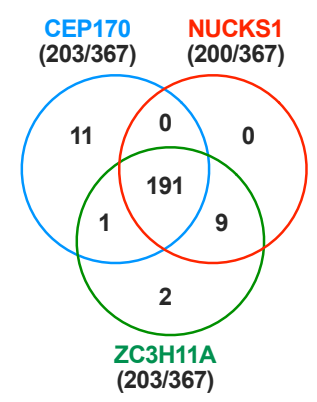

E

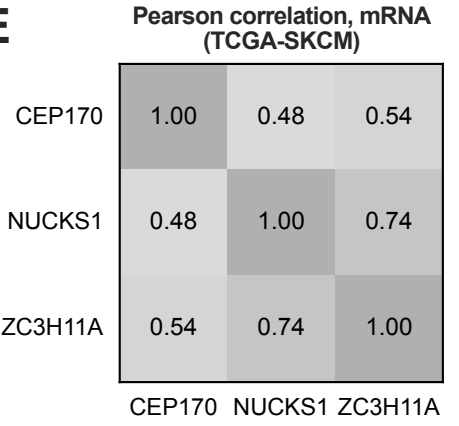

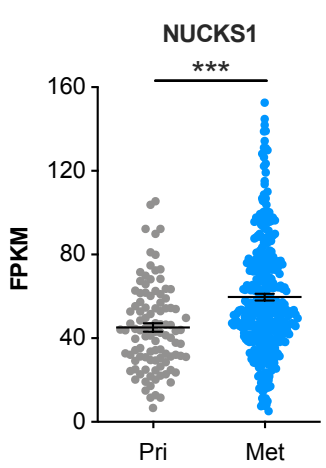

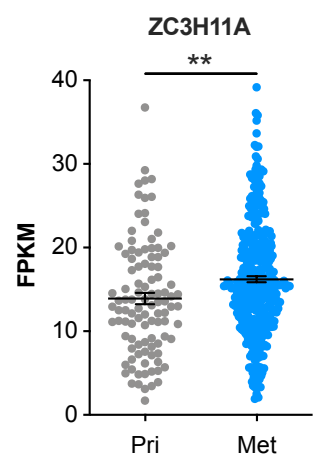

G Enrichment Plot:

Winnepenninckx_Melanoma_Metastasis_Up
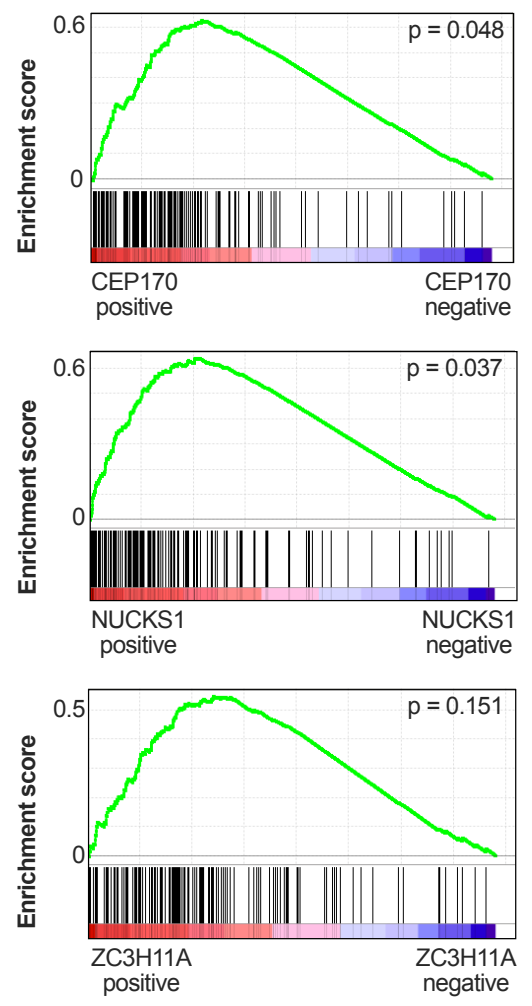

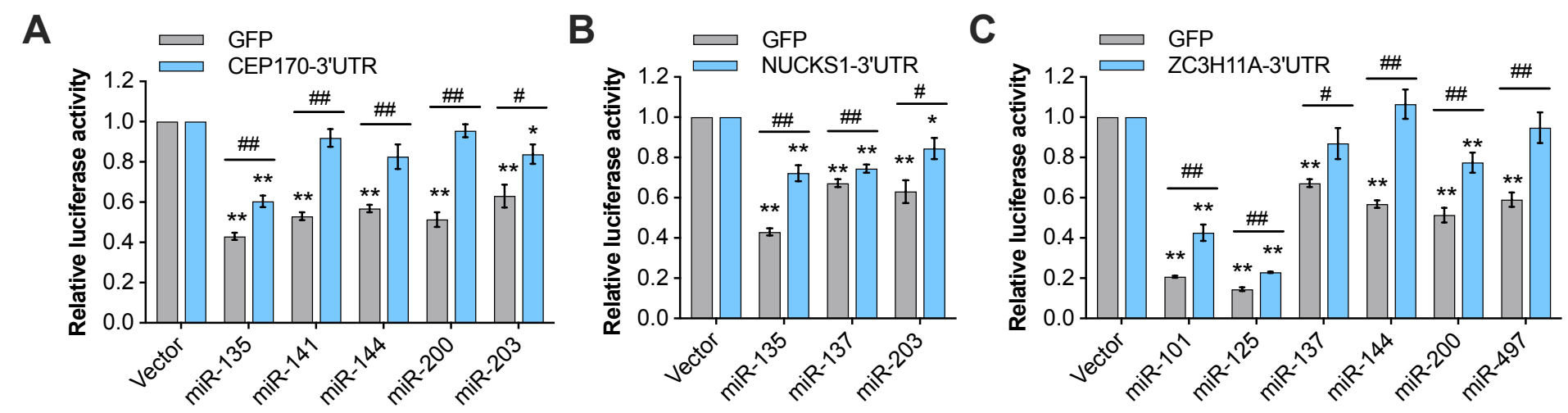

D

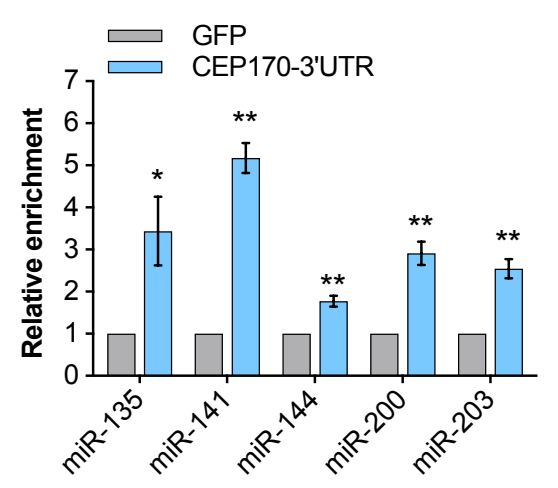

E

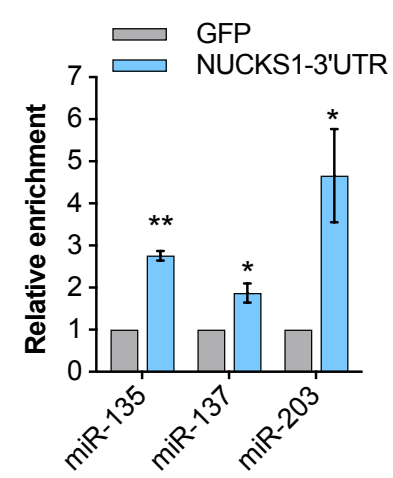

F

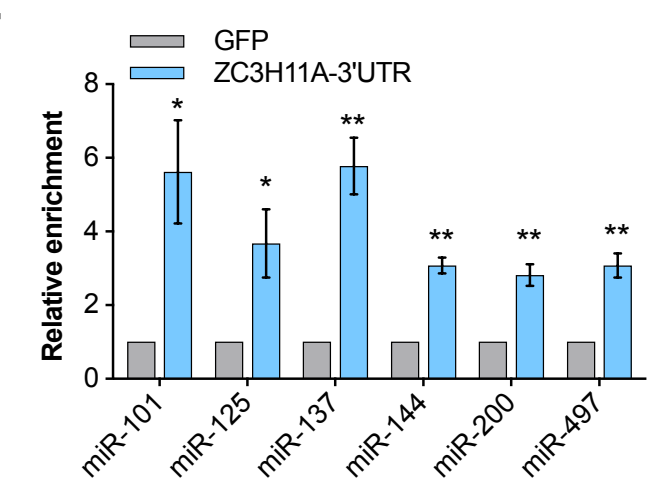

G

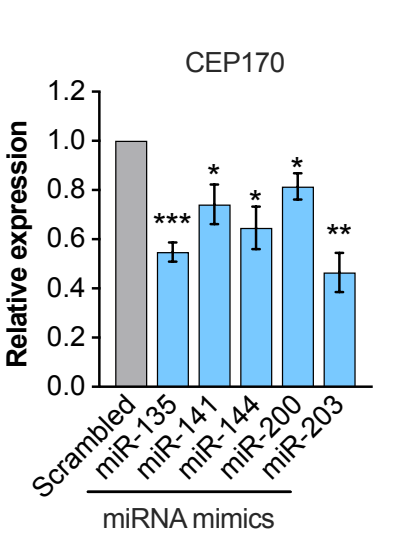

H
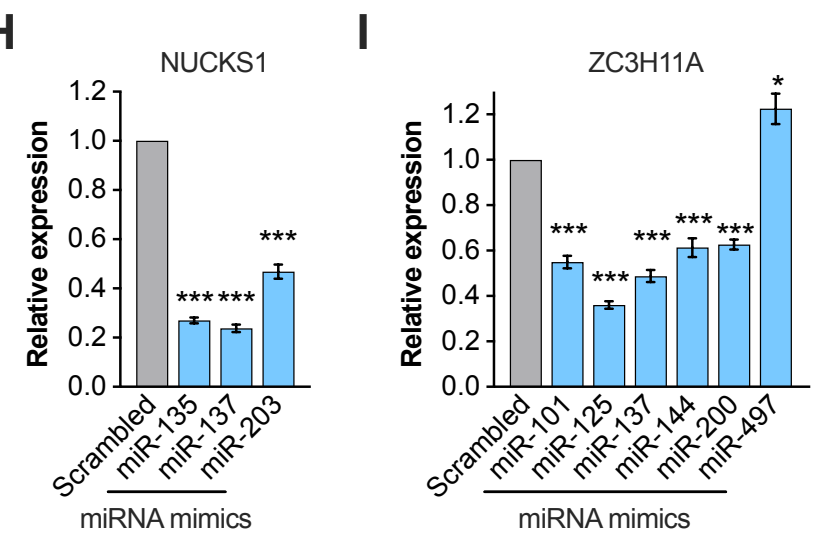

L

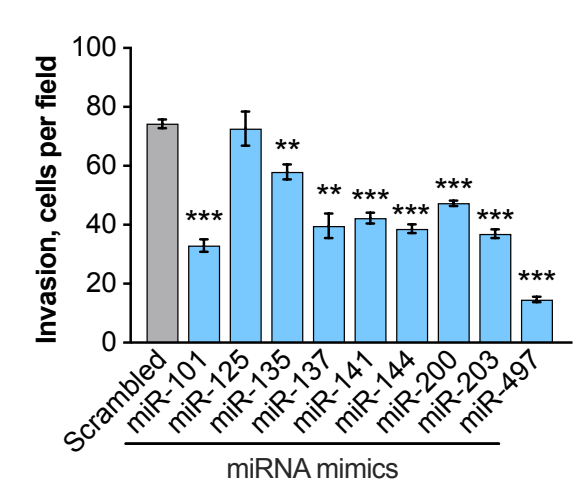

$J$
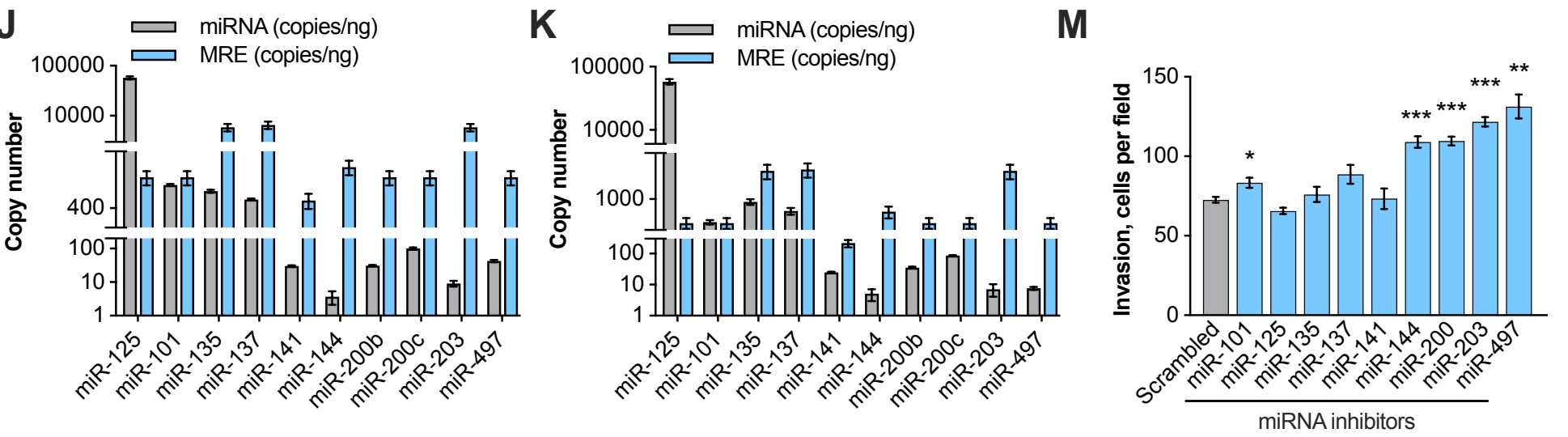

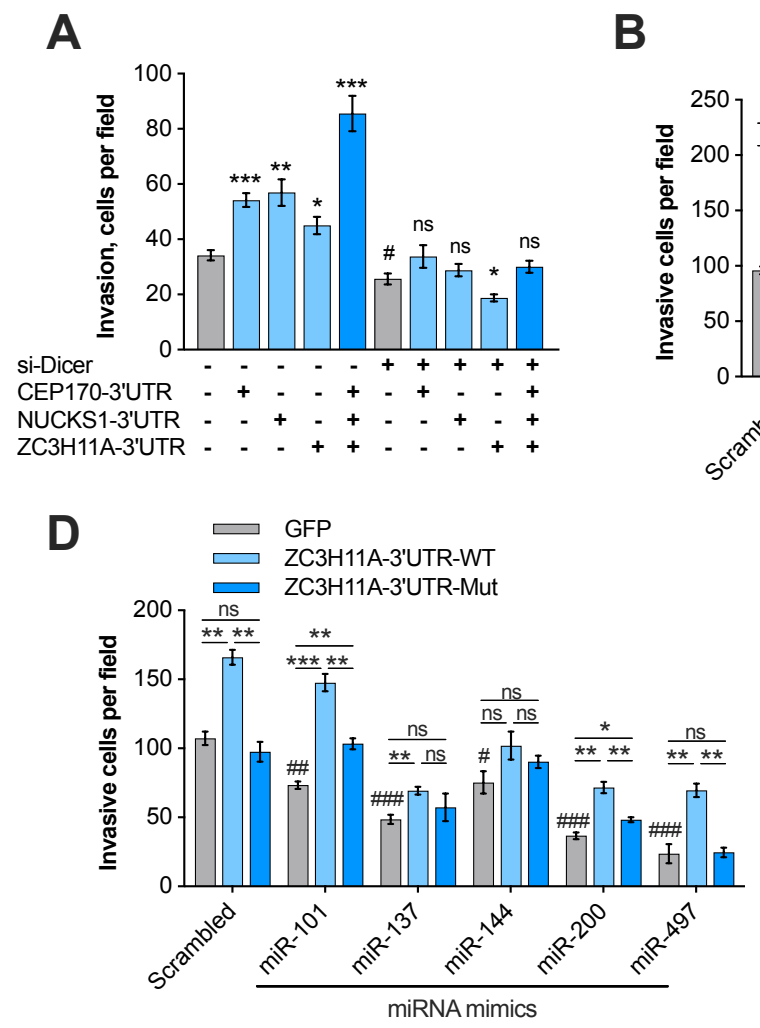
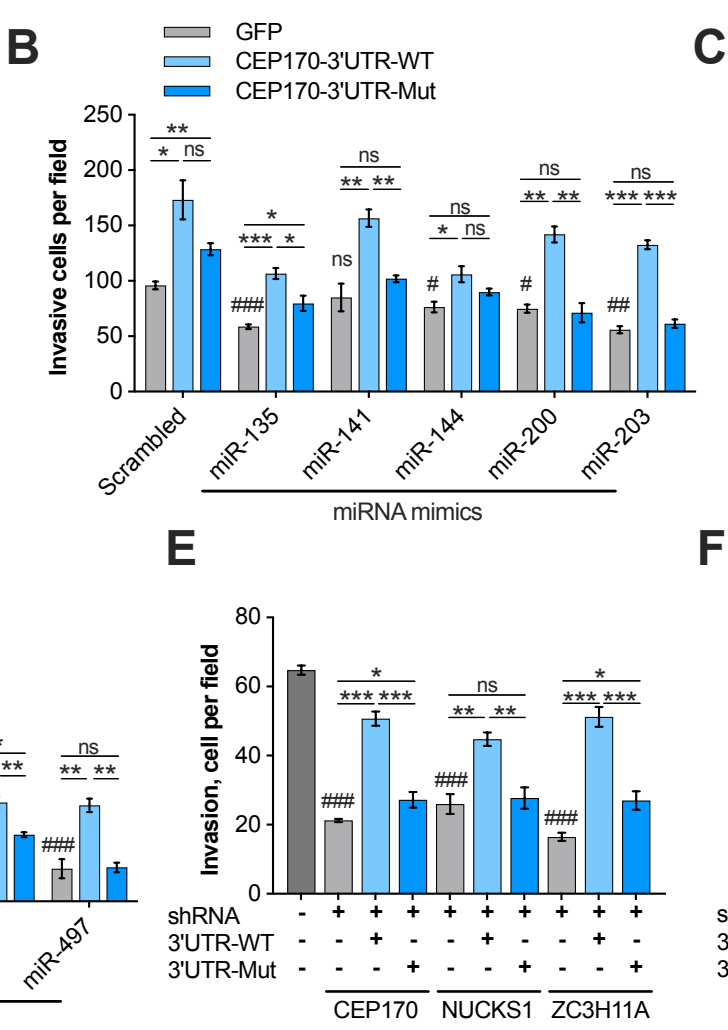

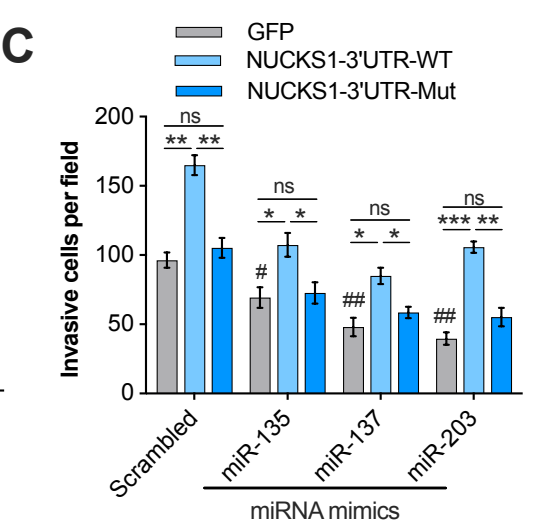

F

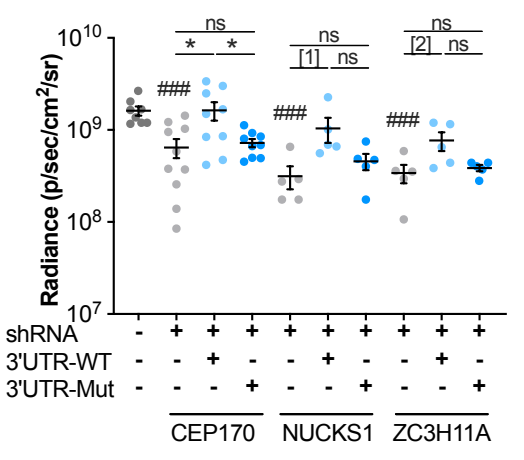

G

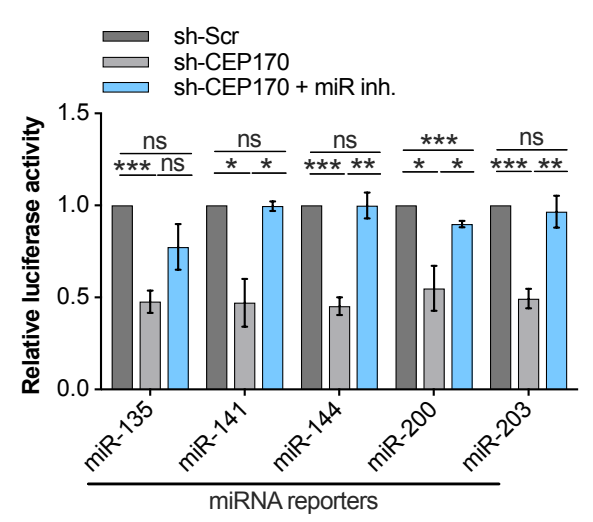

J

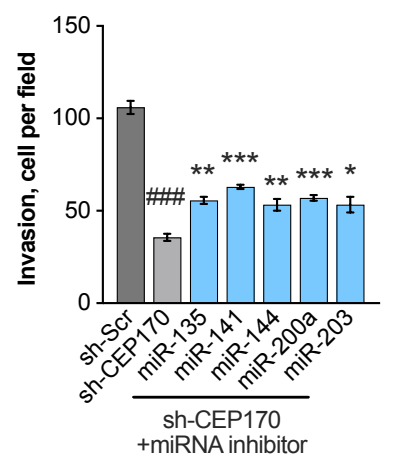

H

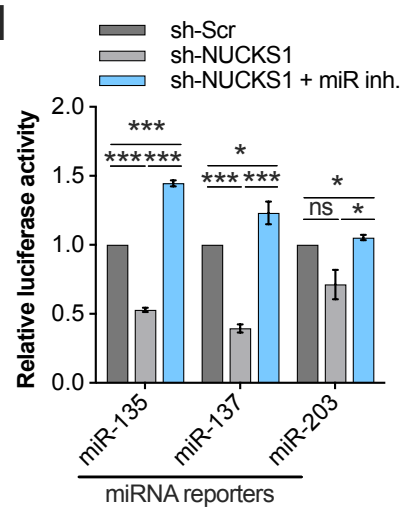

L

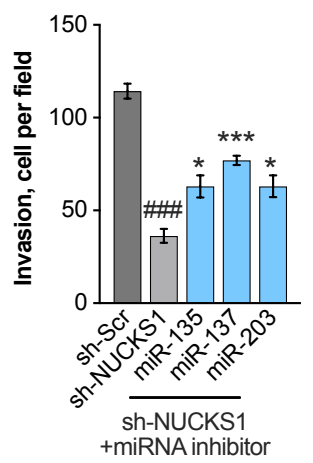

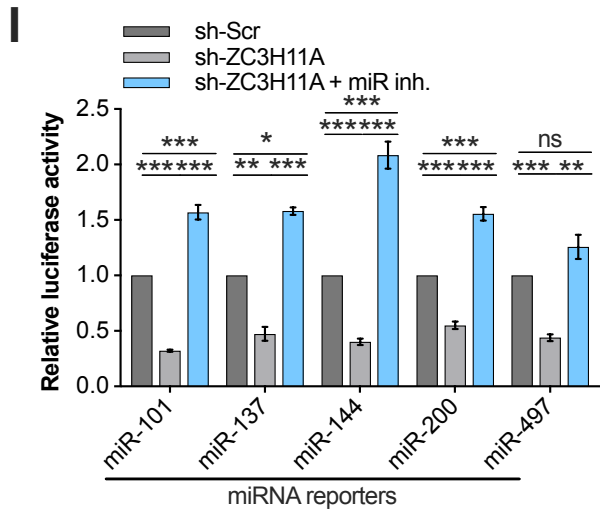

M

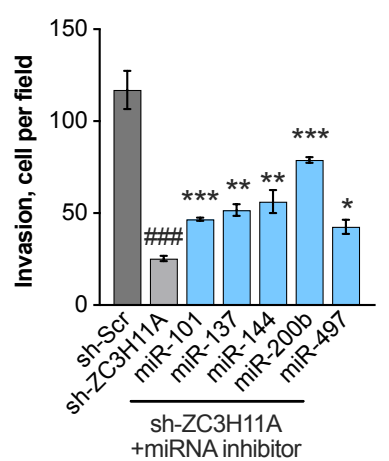

N

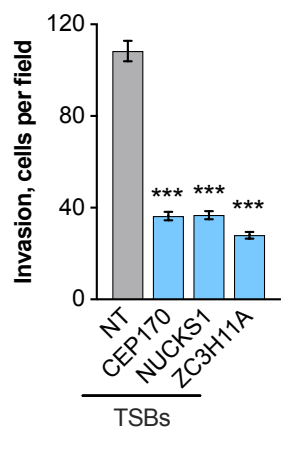

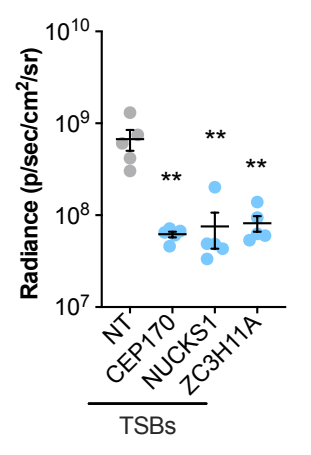


A

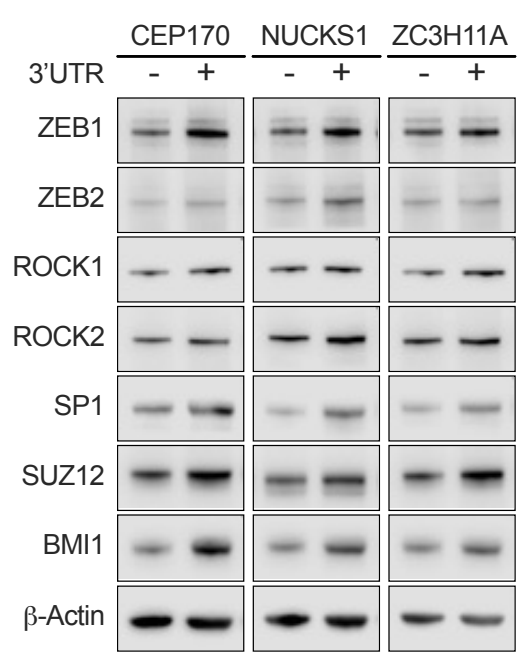

B

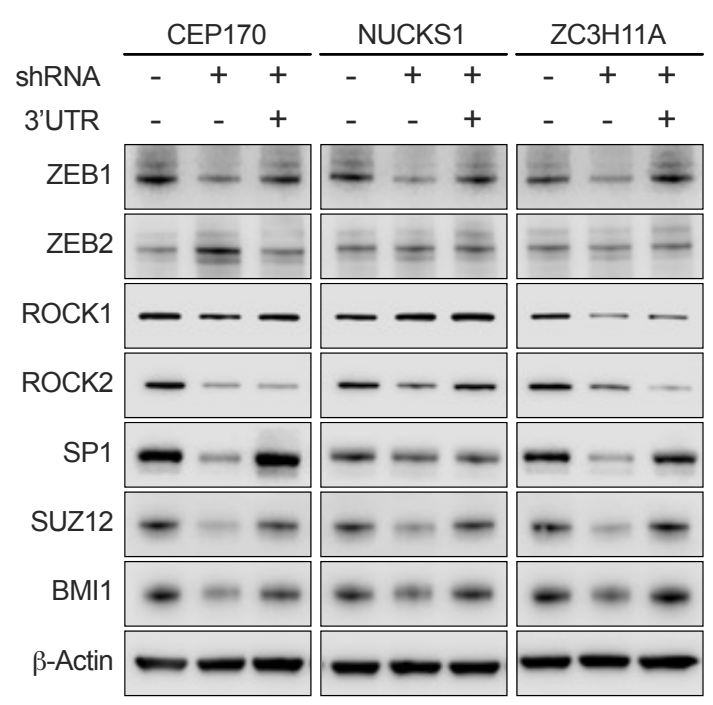

E

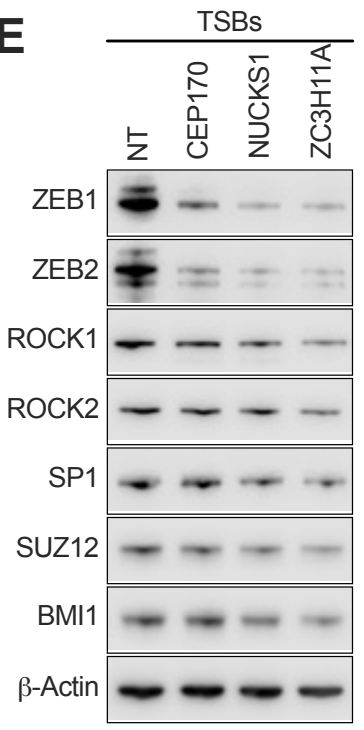

C

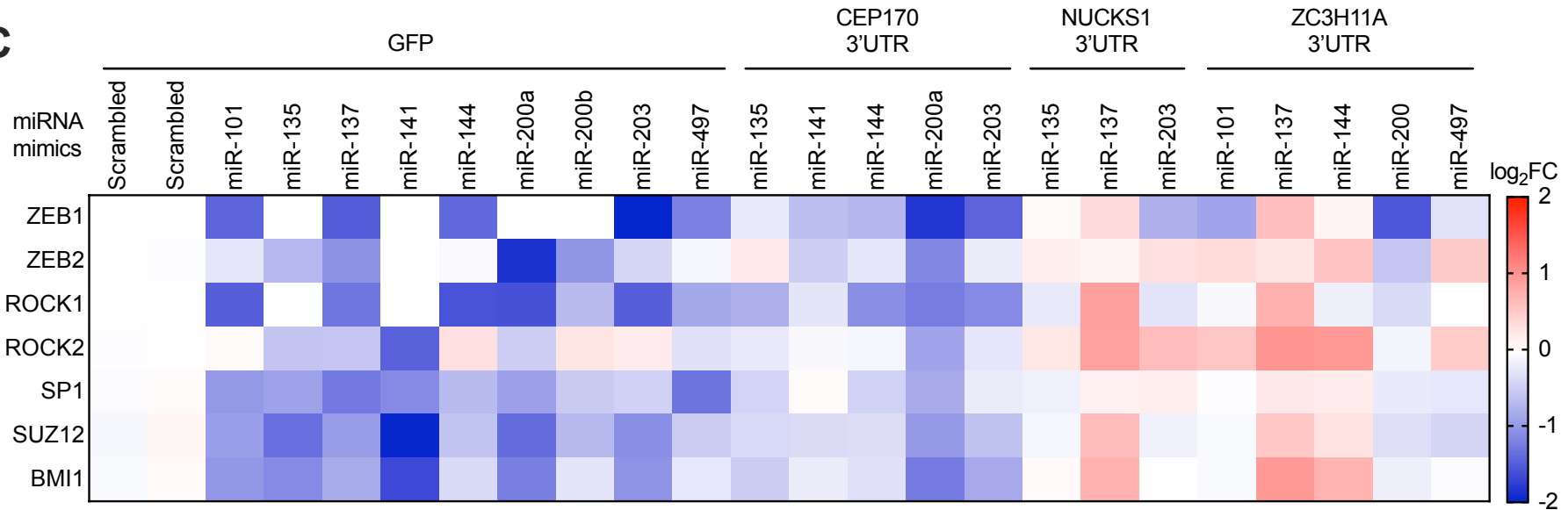

D

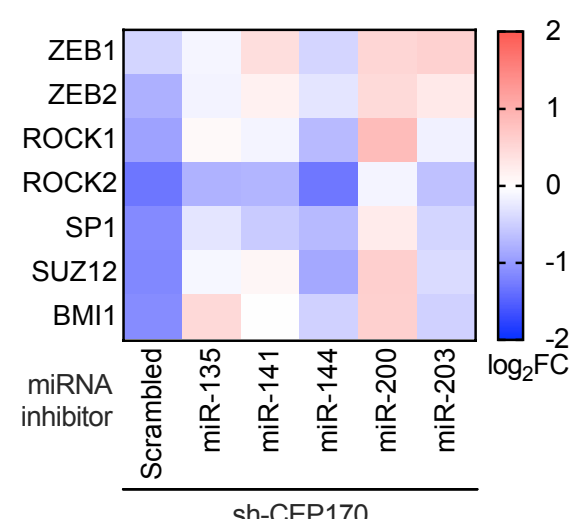

sh-CEP170
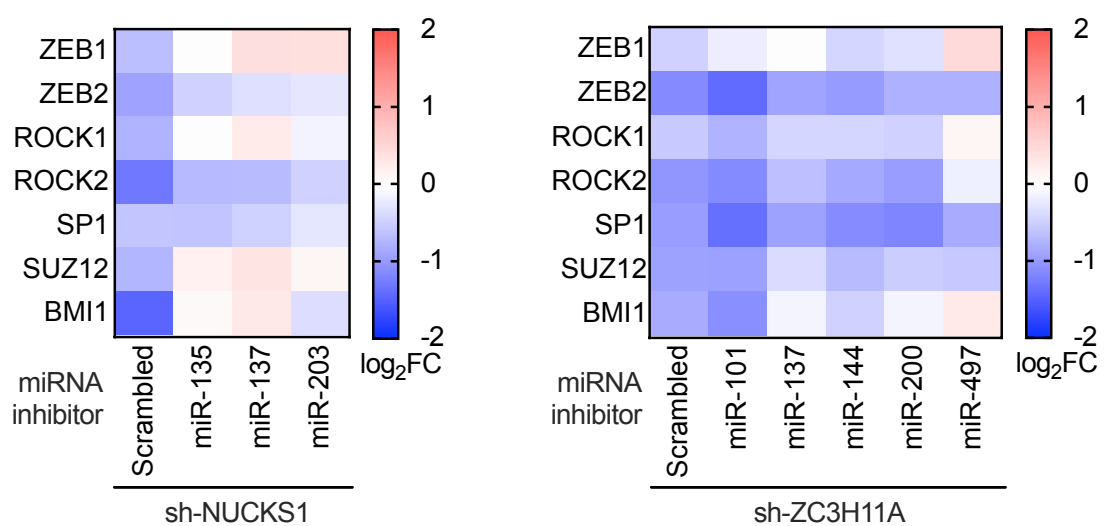
A

TCGA-BRCA

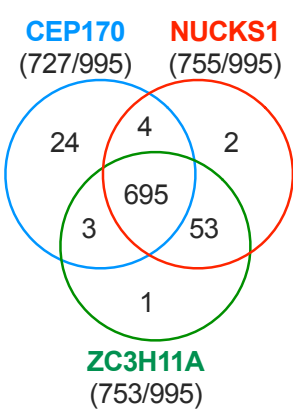

B
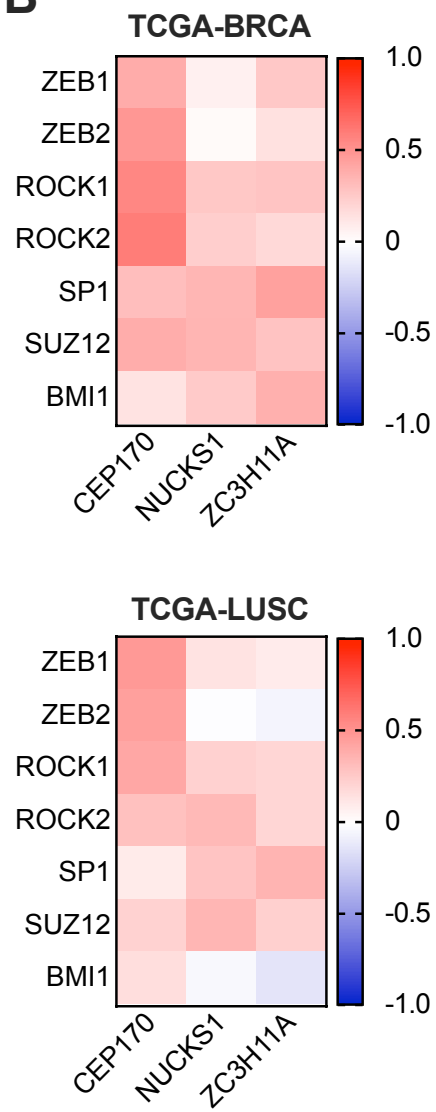

TCGA-COAD
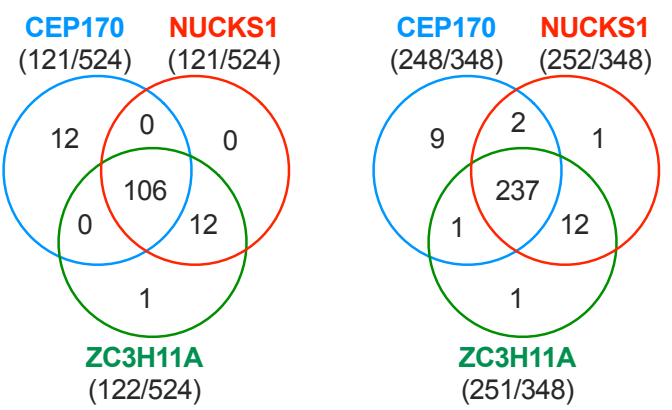

TCGA-LUAD

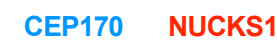

(342/503) (357/503)

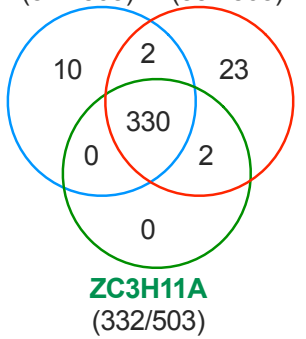

TCGA-LUSC

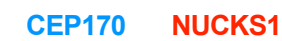

(238/466) (238/466)

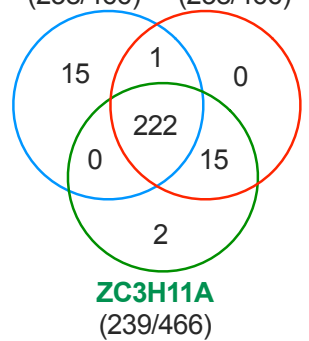

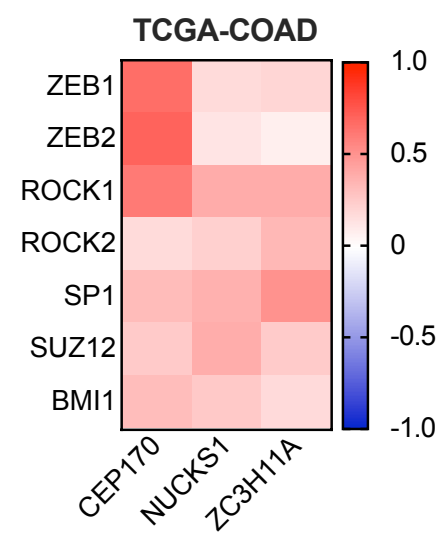
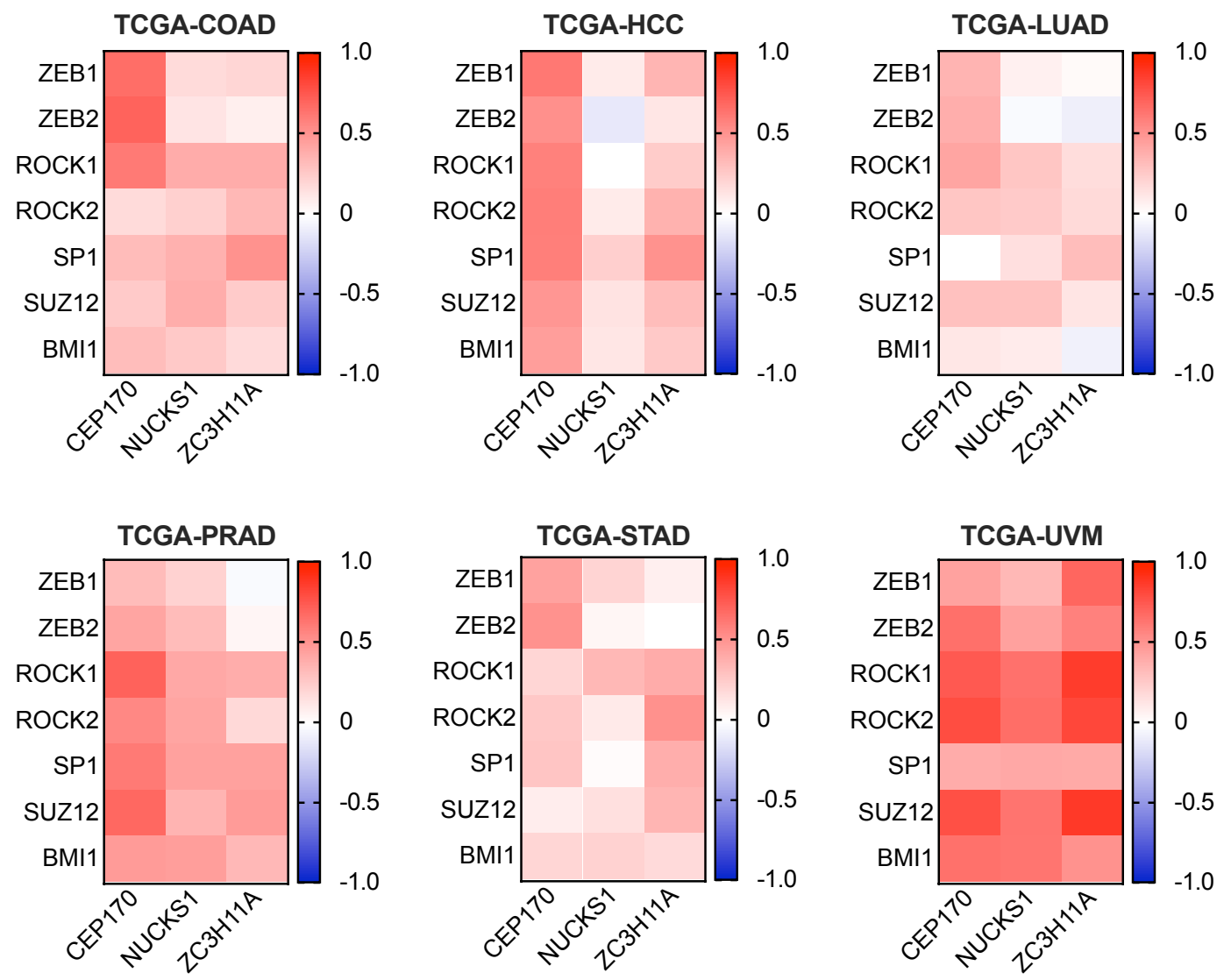
A
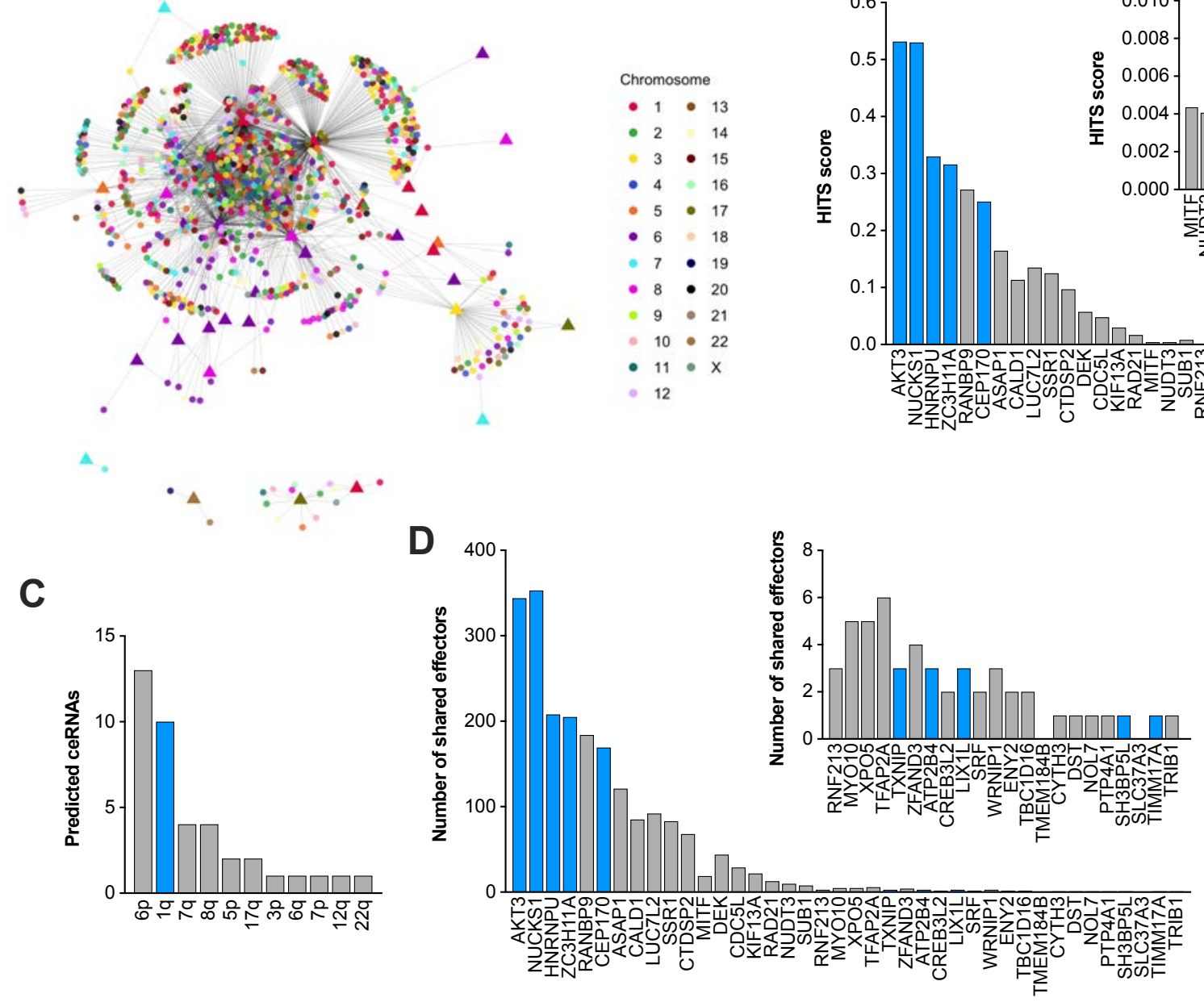

E

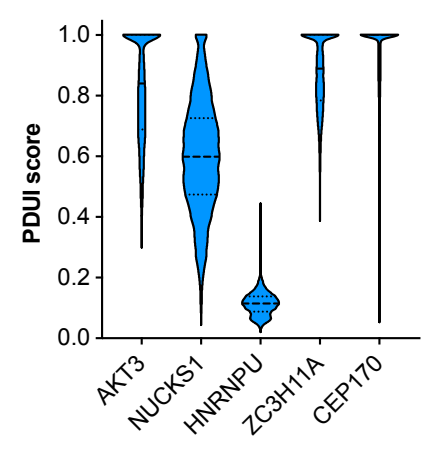

F

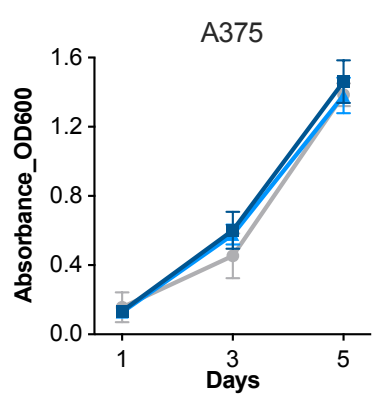

H

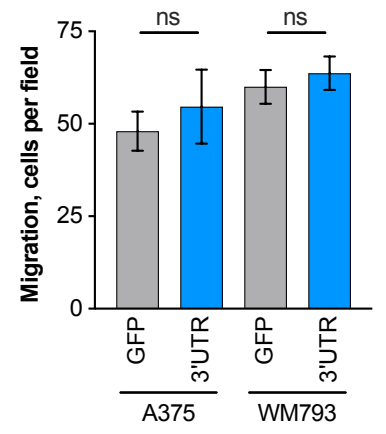

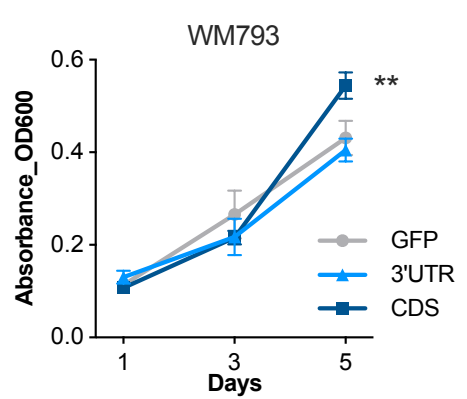

G
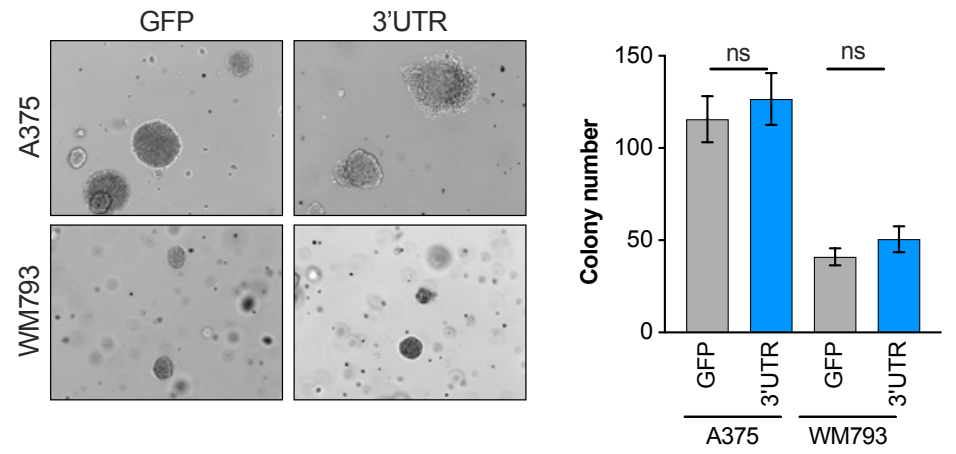
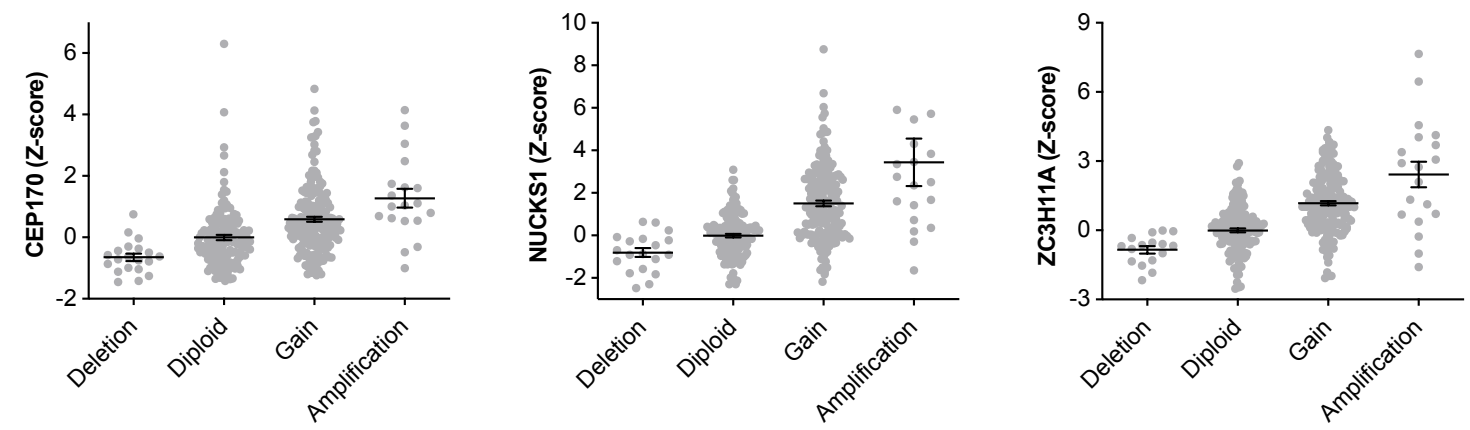


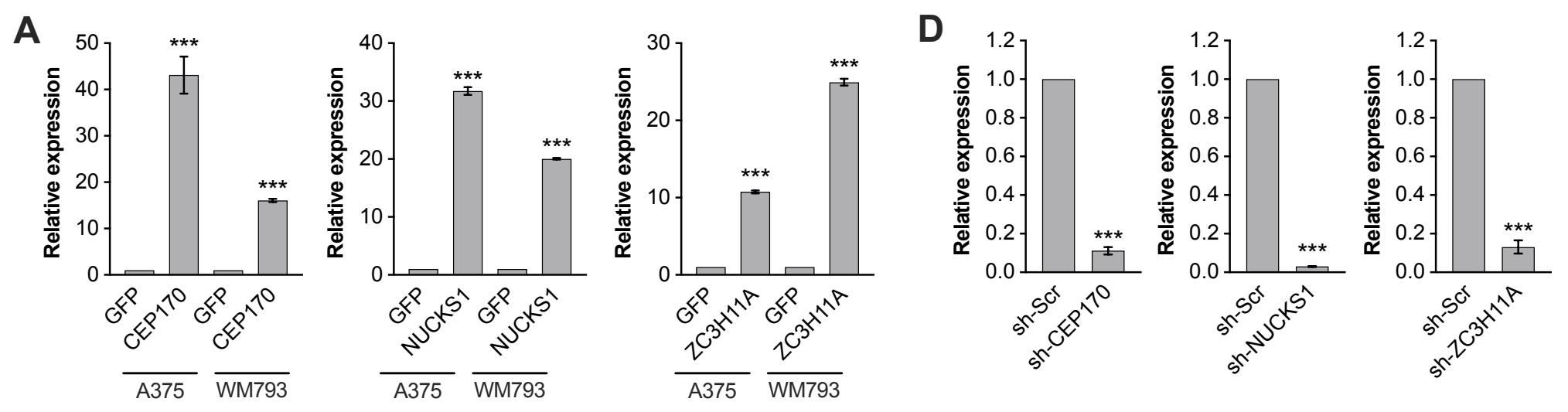

B
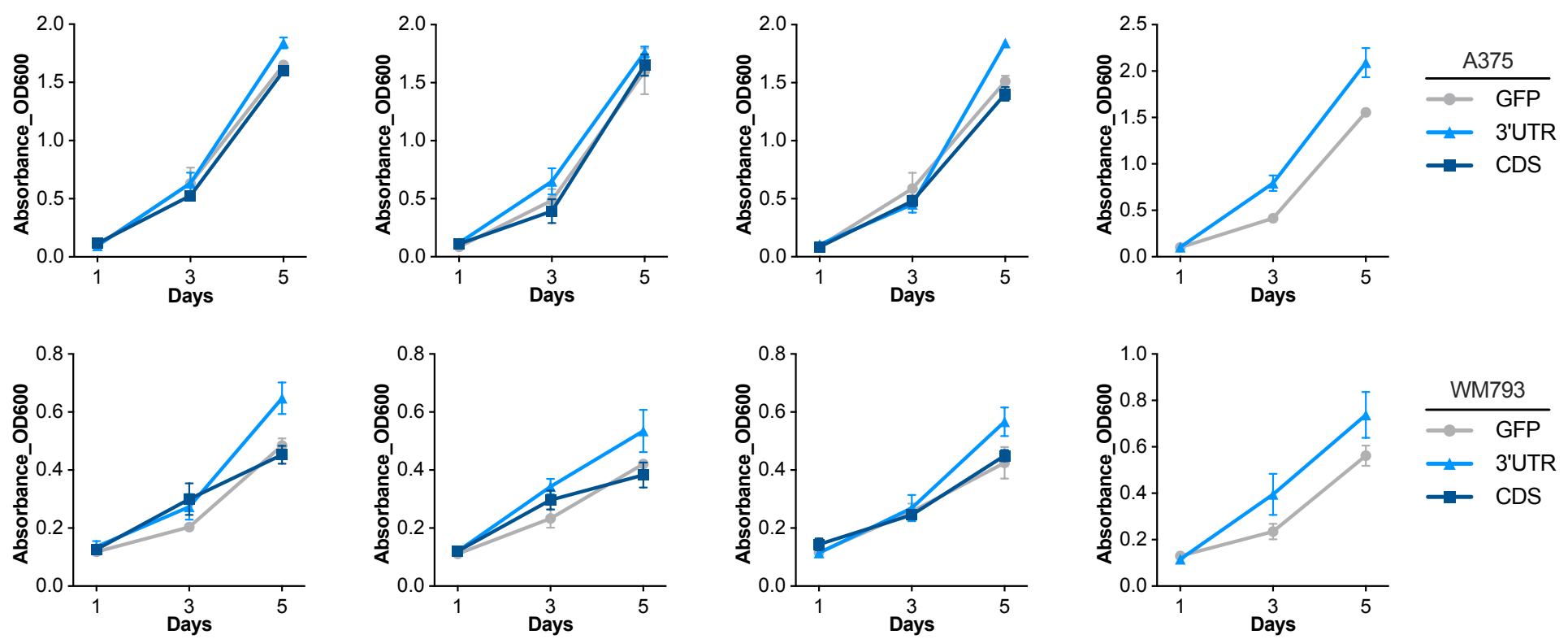

E

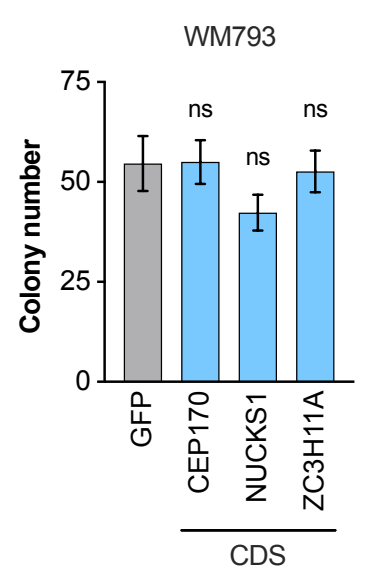

F
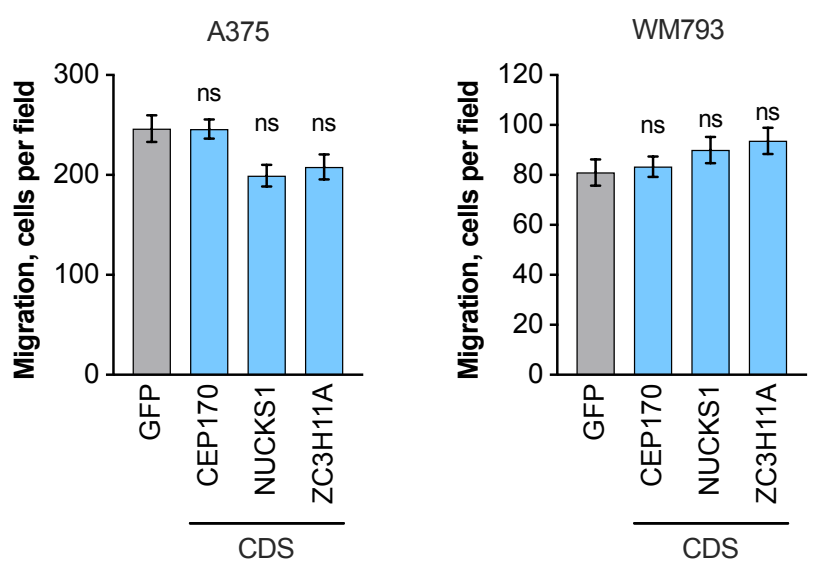


\section{Xu et al., Figure S3}

A

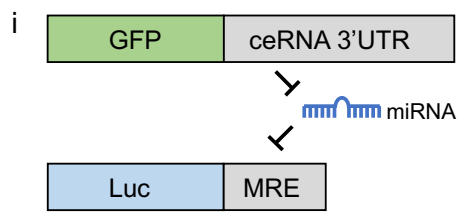

ii Streptavidin beads

Q biotinylated probes miRnAs \begin{tabular}{|c|l}
\hline GFP & ceRNA 3'UTR \\
\hline
\end{tabular}
B

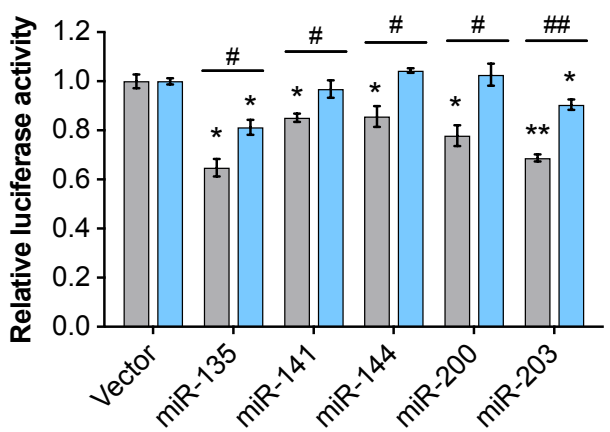

C

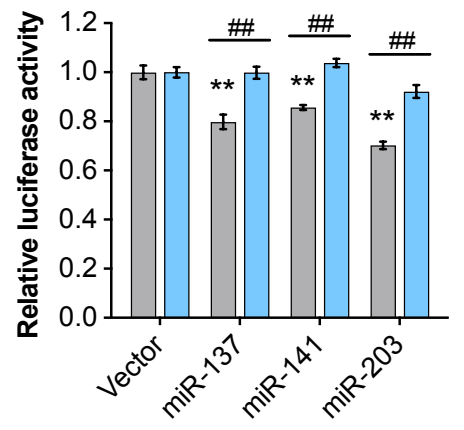

D

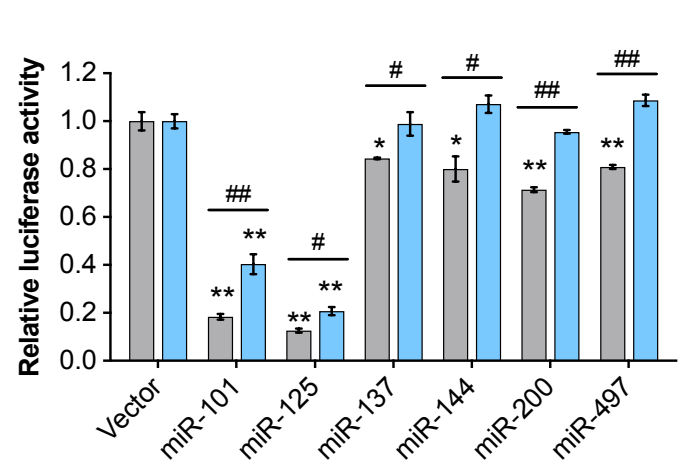

E

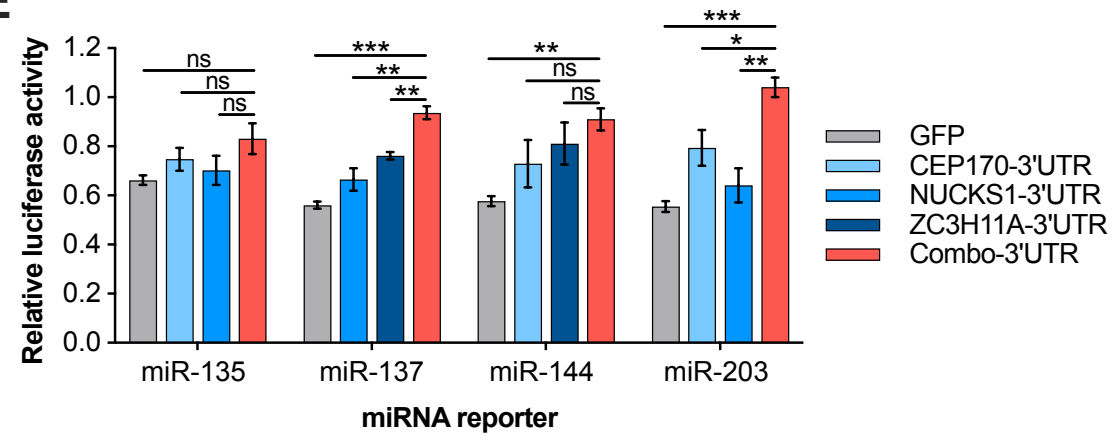

F

A375

G

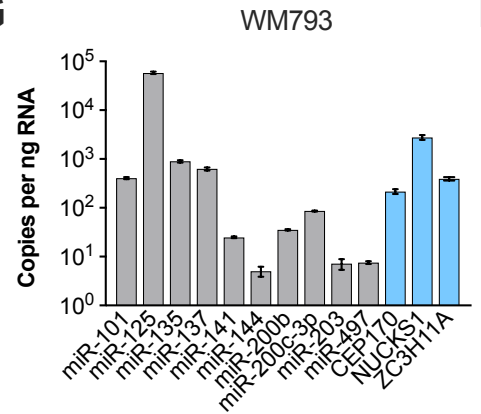

H

1205Lu
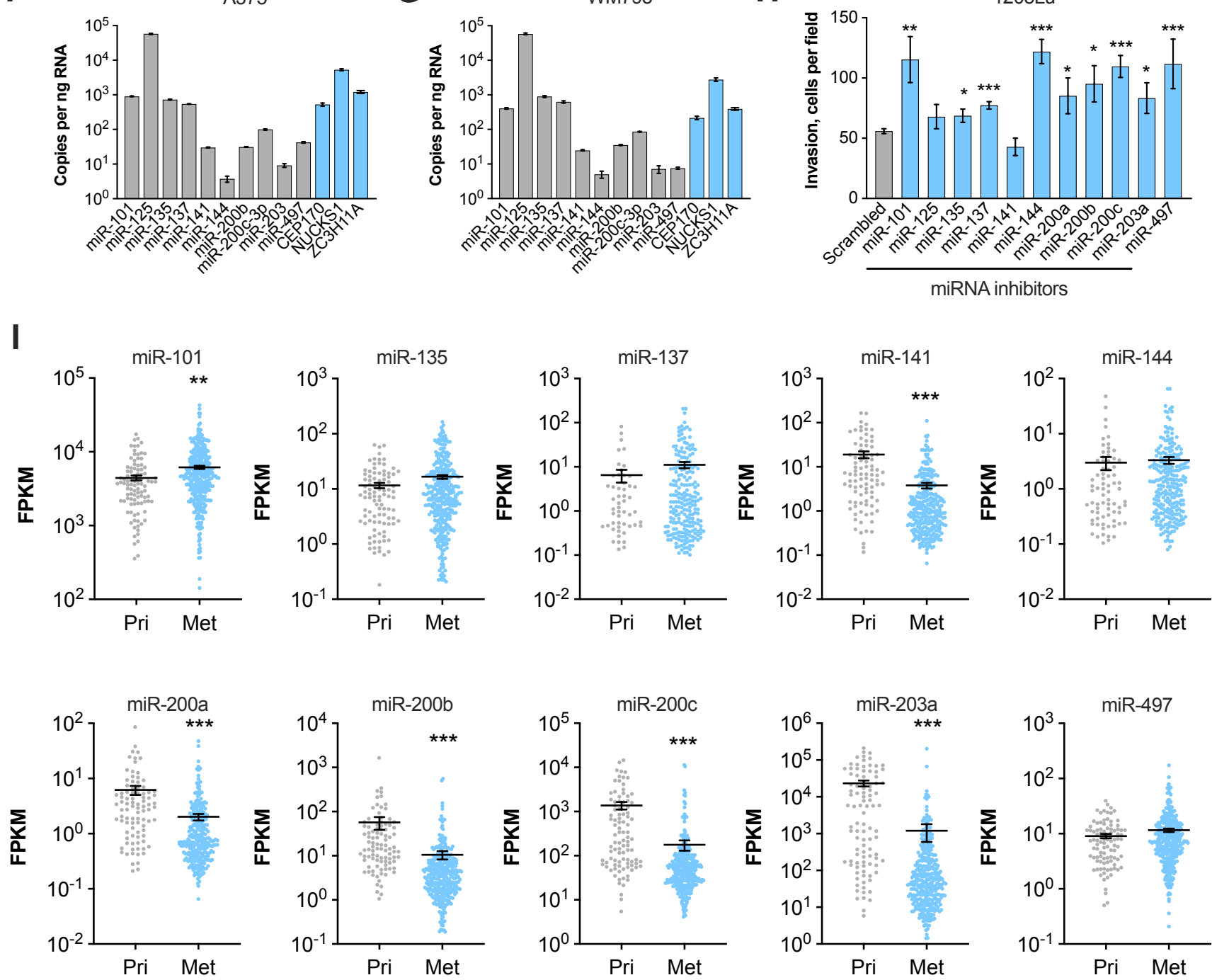
A

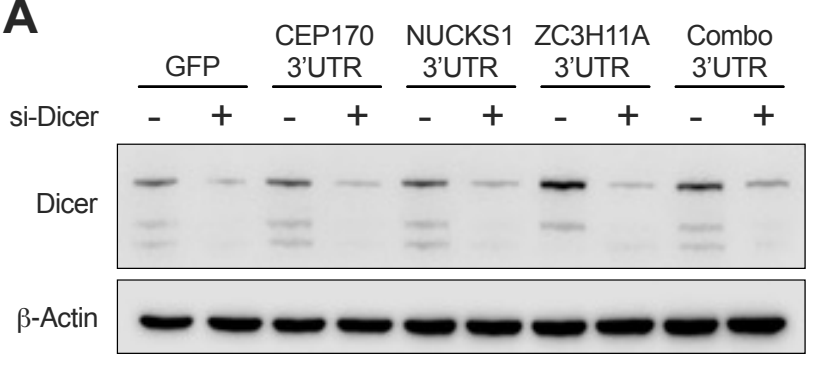

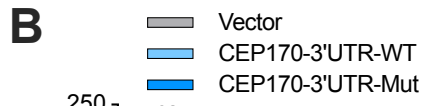

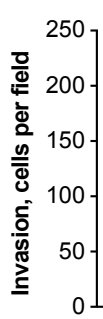
ns CEP170-3'UTR-Mut
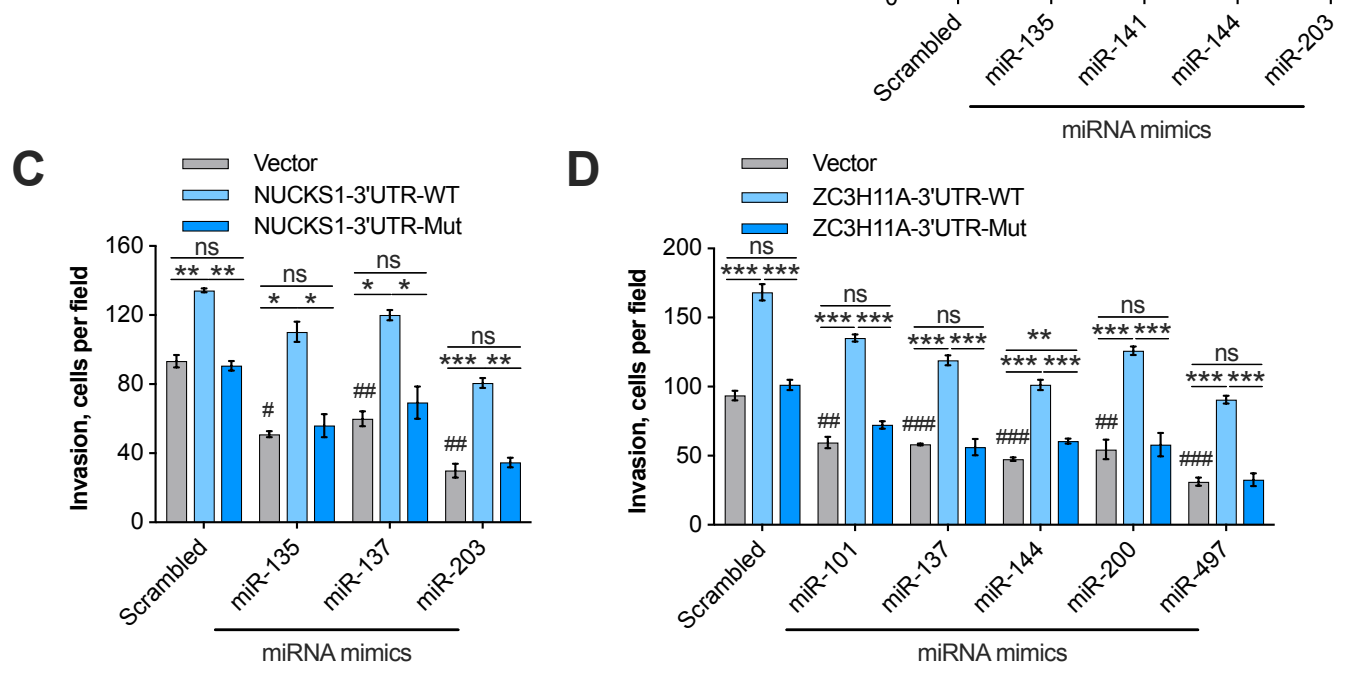

E

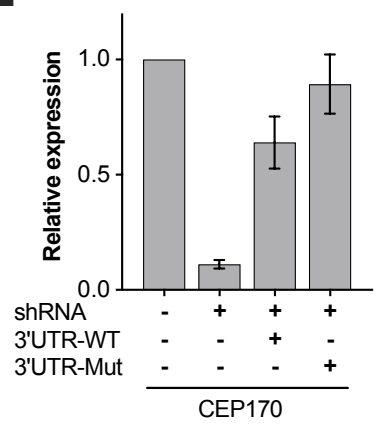

F

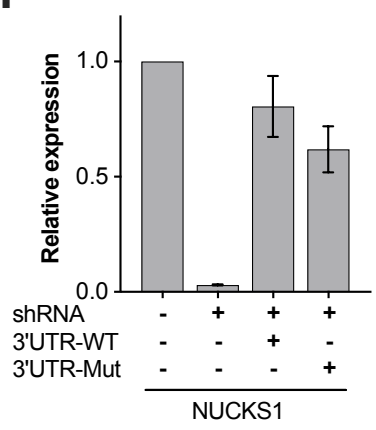

G

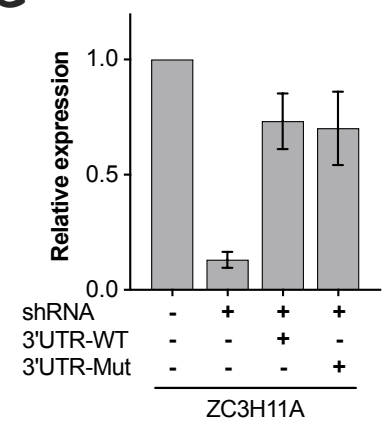

H

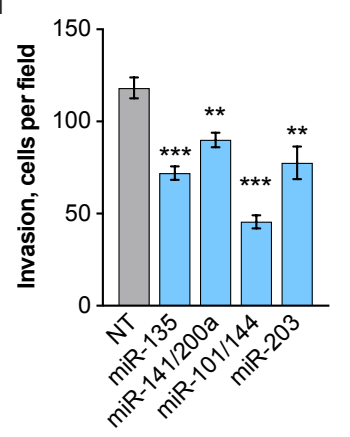

I

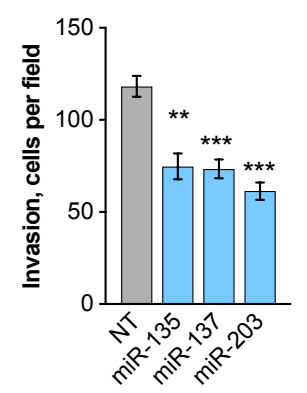

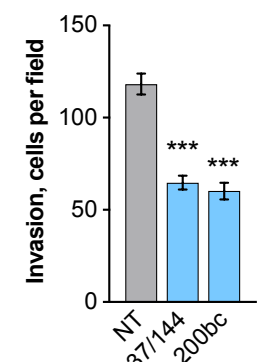


A

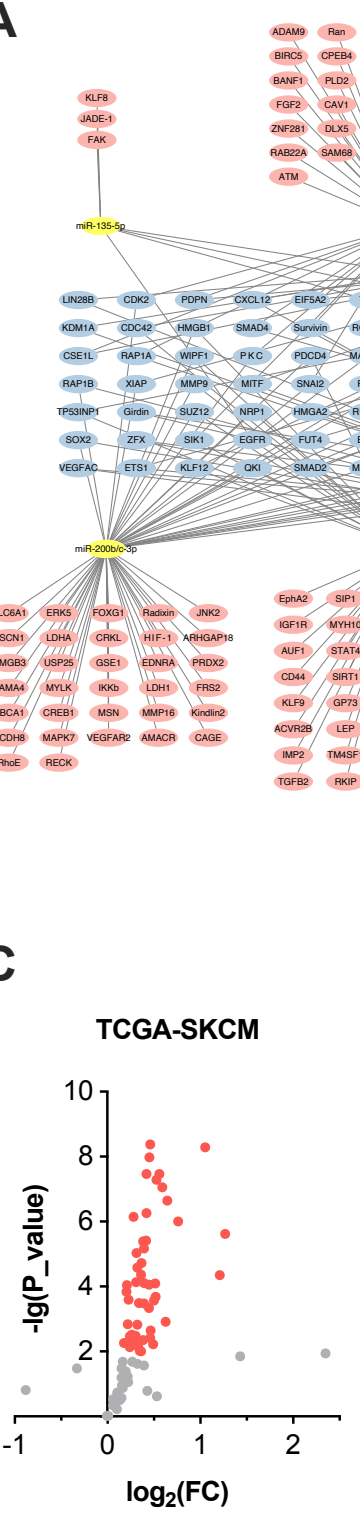

ADAM9 Ran RASAL2 NUAK1 MAT2AB CPERA SPABC SLUG FABPA SAC Amexin A4 GASA1 A SAMEB LASPI POHB Robor 1

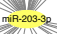

FYYD6 BMP7 CULAA DUSP4 AEG1 YBXX OTUD78 TFAP2C MYO1O PTN RASGRFF TRX3 HEY2 PXN PTPAAS MRGBP GREMt Snail DEL1 YBI SLC22A18 TCF4 CIBP1 FMNL2 2 BTB7 RICTOR ANT2 EDIL3 PAROS
ERBB2 SUUSBH1 PAXI2A GALNTI SET MMP11 MTOH EIFAGEP1 NEU1 NAIFI OKK3 ANGPT2 SIFT MLK3 FOXS1 STAT3 RABBD TSTA3 SMURF, TXNROY PADI2 VDR STARDI3 FUTS ABL2 BTG2 GAB2 SAF1 IL1R CD147 ABL2

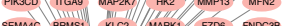
SEMAAG BRMS1 KLC2 MARK1 FRDE FNDCBB HDAC4 GALNTIA KIAAA522 FUT6 NEDDS CREBS

E

potential ceRNA

effectors

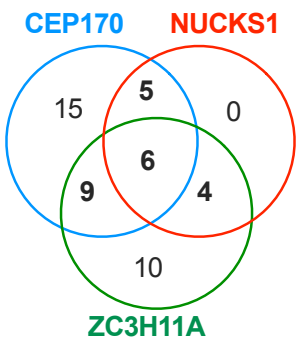

PBX3 ADAMIO $\prod_{\text {CEPS5 PAXB IITAA }}$ GSPT1 KPNAA CCNB1 ADAMTSS TAGLN AP4 MAPKG TOP2A ARIDIA FOSB MAACKS GHHL2 III NOS1 BLACAT LIS1 MAPSKG HOXA10 CLK3 PUAA CO51 SLCMAAS FROT EROIL
B

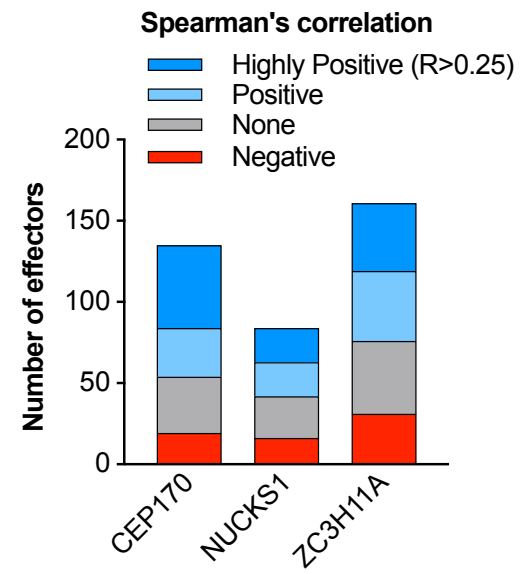

$\mathbf{F}$

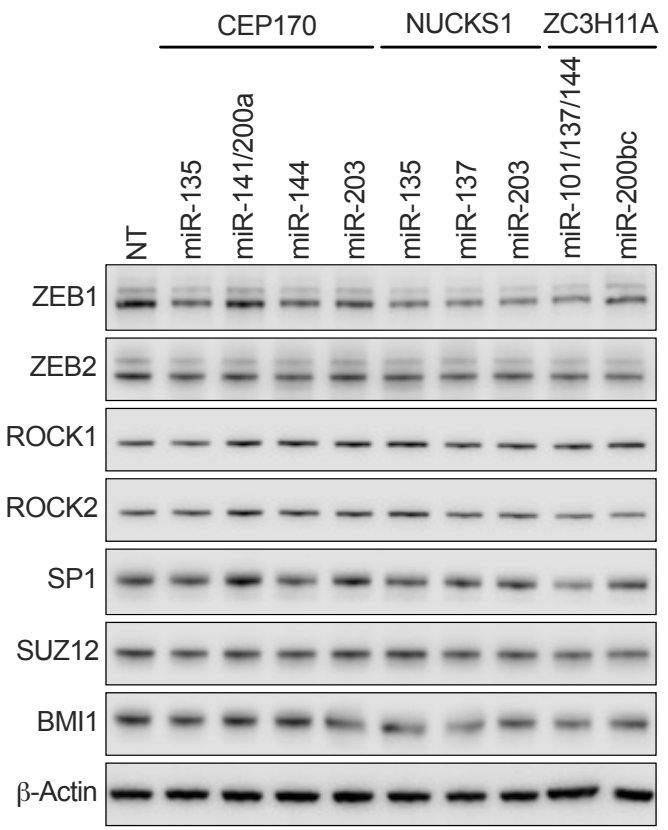

G

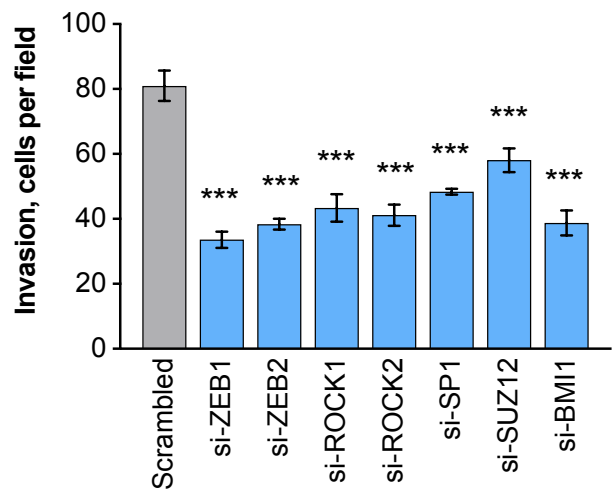

